\title{
s \\ Quantum phase transitions of metals in two spatial dimensions. I. Ising-nematic order
}

\author{
Max A. Metlitski and Subir Sachdev \\ Department of Physics, Harvard University, Cambridge, Massachusetts 02138, USA
}

(Received 28 February 2010; revised manuscript received 18 May 2010; published 16 August 2010)

\begin{abstract}
We present a renormalization-group theory for the onset of Ising-nematic order in a Fermi liquid in two spatial dimensions. This is a quantum phase transition, driven by electron interactions, which spontaneously reduces the point-group symmetry from square to rectangular. The critical point is described by an infinite set of 2+1-dimensional local field theories, labeled by points on the Fermi surface. Each field theory contains a real scalar field representing the Ising order parameter and fermionic fields representing a time-reversed pair of patches on the Fermi surface. We demonstrate that the field theories obey compatibility constraints required by our redundant representation of the underlying degrees of freedom. Scaling forms for the response functions are proposed and supported by computations up to three loops. Extensions of our results to other transitions of two-dimensional Fermi liquids with broken point-group and/or time-reversal symmetry are noted. Our results extend also to the problem of a Fermi surface coupled to a U(1) gauge field.
\end{abstract}

DOI: 10.1103/PhysRevB.82.075127

PACS number(s): 71.10.Hf, 73.43.Nq, 75.10.Kt, 71.27.+a

\section{INTRODUCTION}

A number of recent experiments ${ }^{1-4}$ have noted the presence of Ising-nematic order in the enigmatic normal state of the cuprate superconductors. This order is associated with electronic correlations which spontaneously break the square lattice symmetry to that of a rectangular lattice, i.e., the symmetry of $90^{\circ}$ rotations is lost, and the $x$ and $y$ directions become inequivalent. This broken symmetry is associated with an Ising order parameter, which we will represent below by a real scalar field $\phi$.

Of particular interest are recent experiments on the anisotropy of the Nernst signal ${ }^{4}$ in $\mathrm{YBa}_{2} \mathrm{Cu}_{3} \mathrm{O}_{y}$, which indicate that the Ising-nematic order has its onset at the temperature $T=T^{*}$, which also marks the boundary between the "pseudogap" region and the "strange metal." These results call for the theory of the quantum phase transition involving Ising-nematic ordering in a Fermi-liquid metal. Such a quantum critical point would play an important role in the theory of the strange metal. The metallic Ising-nematic critical point is also of importance in experiments ${ }^{5}$ on $\mathrm{Sr}_{3} \mathrm{Ru}_{2} \mathrm{O}_{7}$, where the observations of resistance anisotropies have demonstrated spontaneous Ising-nematic ordering. Finally, there are clear indications of Ising-nematic order driven by electron correlations in the pnictides. ${ }^{6-9}$

One approach to the Ising-nematic ordering is to take a liquid-crystalline perspective ${ }^{10}$ and view it among a class of phases with broken square lattice symmetry. ${ }^{11-14}$ Isingnematic phases are also a generic feature of frustrated and doped antiferromagnets because the Ising-nematic order survives after antiferromagnetism [at wave vectors $\neq(\pi, \pi)$ ] has been disrupted by thermal ${ }^{15,16}$ or quantum ${ }^{17,18}$ fluctuations.

A complementary point of view ${ }^{19-31}$ is to start from the Fermi liquid with perfect square lattice symmetry and look for the Pomeranchuk instability of Landau's Fermi-liquid theory in the angular momentum $\ell=2$ channel. Almost all of these works rely on the perspective of Hertz, ${ }^{32}$ in which the electrons are integrated out to yield a Landau-damped effective action for the scalar order parameter $\phi$; the low-energy particle-hole excitations near the Fermi surface lead to longrange interactions in the action for $\phi$. However, this procedure of successive integration of fermionic and then bosonic degrees of freedom is clearly dangerous. A systematic renormalization-group (RG) analysis requires that all excitations at a given energy scale be treated together. Consequently, a complete scaling analysis of the Ising-nematic critical point is lacking: such an analysis should be based on a local field theory and provide a scheme for computing the scaling dimensions of all perturbations of the critical point.

We can also consider the onset of Ising-nematic order in a superconductor rather than in a Fermi liquid. In a $s$-wave superconductor, the fermionic excitations are fully gapped, and so the theory for $\phi$ has no long-range interactions: consequently the transition is in the universality class of the 2 +1 -dimensional pure Ising model. A $d$-wave superconductor does have gapless fermionic excitations at special "nodal points" in the Brillouin zone, and these nodal fermions do modify the universality of the transition away from pure Ising. ${ }^{33,34}$ A fairly complete understanding of the Isingnematic transition in $d$-wave superconductors has been reached in recent work ${ }^{35,36}$ using a large- $N$ expansion, where $N$ is the number of fermion components.

This paper provides a scaling theory of the Ising-nematic quantum critical point in two-dimensional metals, satisfying the requirements stated above. Our theory builds upon the work in the $d$-wave superconductor, ${ }^{35,36}$ and also on advances by Polchinski, ${ }^{37}$ Altshuler, Ioffe, and Millis, ${ }^{38}$ and Sung-Sik Lee ${ }^{39,40}$ on a closely related problem: the dynamics of a Fermi surface with the fermions coupled minimally to a $\mathrm{U}(1)$ gauge field.

We focus on a pair of time-reversed patches on the Fermi surface and describe their vicinity by a local 2+1-dimensional field theory. In principle, there are separate critical theories for each pair of time-reversed points on the Fermi surface, as is also the case in the Fermi-surface "bosonization" methods. ${ }^{27,28,41-45}$ However, a key difference from the latter methods is that each Fermi-surface point is associated with a 2+1-dimensional theory and not a $1+1$ dimensional theory. This means that there is a redundancy in 
our description and sowing the theories together is not trivial: we show in Sec. IV A how this is done in a consistent manner.

Apart from their application to the Ising-nematic transition of interest, simple extensions of our results apply also to the U(1) gauge field case, and to other symmetry breaking transitions in Fermi liquids involving order parameters which carry momentum $\vec{Q}=0$. We will describe these cases in Sec. II below and briefly indicate the needed extensions in the body of the paper.

Transitions with order parameters which carry momentum $\vec{Q} \neq 0$ lead to different field theories, which will be described in a subsequent paper. ${ }^{46}$ After a discussion of the one loop results in Sec. III, we present our main scaling analysis in Sec. IV. This includes a discussion of Ward identities which strongly constrain the structure of renormalization-group flow. Finally, explicit three loop computations appear in Sec. $\mathrm{V}$ and Appendix B.

\section{MODEL}

We consider quantum phase transitions in metals of electrons $c_{\sigma}(\sigma=\uparrow, \downarrow)$ involving an onset of a real order parameter $\phi(x)$ at wave vector $\vec{Q}=0$. The order parameter is taken to have the same transformation properties under lattice symmetries and time reversal as

$$
O(\vec{x})=\frac{1}{V} \sum_{\vec{q}} \sum_{\vec{k} \sigma} d_{\vec{k} \sigma} c_{\vec{k}-\vec{q} / 2, \sigma}^{\dagger} c_{\vec{k}+\vec{q} / 2, \sigma} e^{i \vec{q} \cdot \vec{x}} .
$$

For definiteness, we consider a system on a square lattice. Then, $\phi$ can describe the following patterns of symmetry breaking:

(1) Breaking of the point-group symmetry with $d_{\vec{k} \uparrow}=d_{\vec{k} \downarrow}$ and $d_{\vec{k} \sigma}=d_{-\vec{k} \sigma}$. In these cases $d_{\vec{k}}$ has either $d_{x^{2}-y^{2}}, d_{x y}$, or $g$-wave symmetry. The Ising-nematic transition of most interest to us here corresponds to the $d_{x^{2}-y^{2}}$ or $d_{x y}$ cases. These cases all belong to one-dimensional representations of the square lattice point group and we will argue that these transitions are all in the same universality class.

(2) Breaking of time-reversal and point-group symmetry with $d_{\vec{k} \uparrow}=d_{\vec{k} \downarrow}$ and $d_{\vec{k} \sigma}=-d_{-\vec{k} \sigma}$. In this case $d_{\vec{k}}$ transforms under the two-dimensional $p$-wave representation and so requires a two component order parameter $\vec{\phi}=\left(\phi_{x}, \phi_{y}\right)$. We will not consider the two-component case explicitly but our results have an immediate generalization to this transition. This case corresponds to the "circulating current" order parameters proposed by Simon and Varma, ${ }^{47}$ as was argued in Refs. 33 and 48.

(3) Breaking of spin-inversion symmetry with $d_{\vec{k} \uparrow}=-d_{\vec{k} \downarrow}$. In this case, $d_{\vec{k}}$ can have either $s$-wave symmetry (Ising ferromagnet), $d$-wave symmetry (Ising spin-nematic) or $g$-wave symmetry. Unlike transitions 1 and 2, which respect the full $\mathrm{SU}(2)$ spin-rotation symmetry, in the present case we assume this symmetry is explicitly broken to a U(1) "easy axis" subgroup.

Notice that in all cases, there is a $Z_{2}$ symmetry (either $\pi / 2$ rotation, reflection, or time reversal) under which $\phi \rightarrow-\phi$. Apart from the above symmetry breaking cases, we will also consider the problem of a Fermi surface minimally coupled to a U(1) gauge field. ${ }^{37-40,49-58}$ This case is similar to case 2 above, as we describe below Eq. (2.4). Such models arise in theories $^{57,58}$ of certain $\mathrm{U}(1)$ spin-liquid phases in which $c_{\sigma}$ describe the fermionic spinons. We will therefore refer to this model as the "spin-liquid" case below. The same theory also describes ${ }^{59-61}$ "algebraic charge liquids" in which case the $c_{\sigma}$ are spinless, charge $-e$ fermions, and $\sigma$ represents the charge of the fermion under the emergent $\mathrm{U}(1)$ gauge field; we will not refer to this case explicitly below.

Given the order parameter in Eq. (2.1), we may write down an effective space-time Lagrangian describing the interactions of the order parameter $\phi$ with the fermions as

$$
L=c_{\sigma}^{\dagger}\left[\partial_{\tau}+\epsilon(-i \nabla)\right] c_{\sigma}-O(x) \phi(x)+\frac{1}{2}(\nabla \phi)^{2}+\frac{r_{0}}{2} \phi^{2} .
$$

Here, we have added by hand a gradient term and a mass for the bosonic mode $\phi$. Such terms will be generated automatically after integrating out the high-energy fermions. The absence of higher-order terms in $\phi$ and gradients of $\phi$ will be justified below.

The Lagrangian $L$ in Eq. (2.2) is not yet in a form suitable for our analysis of quantum criticality. The main point is that the fermion spectrum $\epsilon(\vec{k})$ has zeros along the entire Fermi surface of large momenta $\vec{k}$ : so, as is well known, we are not in a position to make a low momentum expansion needed for a field theory. One strategy is to use the Hertz approach ${ }^{32}$ of integrating out all the $c$ fermions to obtain a nonlocal effective action for the order parameter $\phi$. The latter is singular only at small momenta $\vec{q}$ and $\omega$, and so it is then at least permissible to make a low momentum and frequency expansion. However, the terms in the effective for $\phi$ turn out to be highly singular as $\vec{q} \rightarrow 0$ (see Ref. 26 and Appendix A). Moreover, in $d=2$, the strength of the singularity increases with increasing powers of $\phi$ in the effective action. The situation now seems hopeless but progress becomes possible after a key observation: the leading singularities in the $\phi$ effective action appear only when all the $\phi$ fields have their momenta nearly collinear to each other, as is explained in Appendix A, and as will become clear from the structure of our analysis below (by nearly collinear we mean that the angle $\theta$ between the momenta is of order $\left.\theta \sim|\vec{q}| / k_{F}\right)$. In other words, if we are interested only in leading critical behavior, $\phi$ fields with noncollinear momenta effectively decouple from each other. The couplings between $\phi$ fields with noncollinear momenta are then irrelevant corrections to the critical theory. The argument supporting this statement is presented in Appendix A. More generally, consider an $n$-point function

$$
\left\langle\phi\left(\vec{q}_{1}\right) \phi\left(\vec{q}_{2}\right) \phi\left(\vec{q}_{3}\right) \cdots \phi\left(\vec{q}_{n}\right)\right\rangle .
$$

If, as claimed by Hertz, ${ }^{32}$ the field $\phi$ is described by a Gaussian theory, such a correlator would decouple into products over pairs of momenta which sum to zero. However, such a decoupling is too drastic: rather, the decoupling is only over sets of momenta which are collinear with each other, so that 


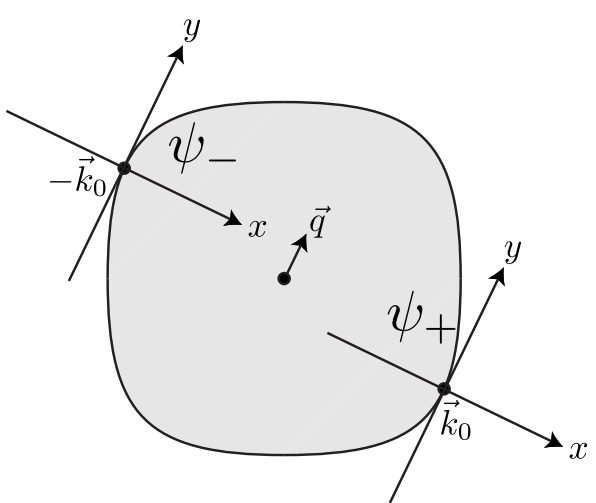

FIG. 1. The shaded region represents the occupied states inside a Fermi surface. Fluctuations of the order parameter $\phi$ at wave vectors parallel to $\vec{q}$ couple most strongly to fermions near the Fermi-surface points $\pm \vec{k}_{0}$. These fermions are denoted $\psi_{ \pm}$.

the leading critical singularity of the above correlator takes the form

$$
\prod_{a}\left\langle\phi\left(\vec{q}_{a 1}\right) \phi\left(\vec{q}_{a 2}\right) \cdots\right\rangle .
$$

Here all the momenta $\vec{q}_{a i}$ in a group $Q_{a}$ are collinear to each other while being noncollinear to momenta in groups $Q_{b}$ with $b \neq a$. We can therefore limit ourselves to $\phi$ fields with momenta along a fixed direction $\vec{q}$. We will now argue that for each such direction $\vec{q}$, there is a sensible and powerful continuum limit of Eq. (2.2).

It is now clear that we may restrict our search for a field theory to that describing the singularities in the $\phi$ correlations for a single group of collinear momenta $Q_{a}$. So let us pick a direction $\vec{q}$ for $\phi$. It is believed that a bosonic mode with momentum $\vec{q}$ interacts most strongly with the patches of the Fermi surface to which it is tangent. ${ }^{37-40}$ Assuming that only a single Fermi surface is present, for each $\vec{q}$ there will be two such points with opposite Fermi-momenta $\vec{k}_{0}$ and $-\vec{k}_{0}$, see Fig. 1. We will denote fermions at these momenta as $\psi_{+}$ and $\psi_{-}$,

$$
\psi_{+\sigma}(\vec{k})=c_{\vec{k}_{0}+\vec{k}, \sigma}, \quad \psi_{-\sigma}(\vec{k})=c_{-\vec{k}_{0}+\vec{k}, \sigma} .
$$

We choose coordinate vectors $\hat{x}$ and $\hat{y}$ to be, respectively, perpendicular and parallel to $\vec{q}$. Then, expanding the fermion energy near $\vec{k}_{0}$ and $-\vec{k}_{0}$, the needed low energy, continuum Lagrangian becomes

$$
\begin{aligned}
L_{k_{0}}= & \psi_{+\sigma}^{\dagger}\left(\partial_{\tau}-i v_{F} \partial_{x}-\frac{1}{2 m} \partial_{y}^{2}\right) \psi_{+\sigma} \\
& +\psi_{-\sigma}^{\dagger}\left(\partial_{\tau}+i v_{F} \partial_{x}-\frac{1}{2 m} \partial_{y}^{2}\right) \psi_{-\sigma}-d_{+\sigma} \phi \psi_{+\sigma}^{\dagger} \psi_{+\sigma} \\
& -d_{-\sigma} \phi \psi_{-\sigma}^{\dagger} \psi_{-\sigma}+\frac{1}{2}\left(\partial_{y} \phi\right)^{2}+\frac{r_{0}}{2} \phi^{2} .
\end{aligned}
$$

Here $v_{F}$ is the Fermi velocity and $m v_{F}$ the Fermi surface curvature radius at $k_{0}$, while $d_{ \pm \sigma}=d_{ \pm k_{0} \sigma}$, and we have added a subscript $k_{0}$ to $L$ to emphasize that this is the Lagrangian for the patch near $\pm \vec{k}_{0}$.
We should stress here that all the fields in Eq. (2.4) are $2+1$-dimensional quantum fields with full dependence upon $x, y$, and $\tau$, i.e., the fields are $\phi(x, y, \tau)$ and $\psi_{ \pm \sigma}(x, y, \tau)$. In principle, we should also add a term $\left(\partial_{x} \phi\right)^{2}$ to Eq. (2.4); however, we omit it at the outset because it will later be seen to be irrelevant near criticality. Further, because of this full dependence on $x$ and $y$, the fermion fields $\psi_{ \pm \sigma}$ describe an extended patch of the Fermi surface near the points $\pm \vec{k}_{0}$, and not just the two points $\pm \vec{k}_{0}$. We place some finite cutoff $\Lambda$ on the size of this patch and will be interested in the scaling behavior at momenta much smaller than this cutoff.

We now discuss the structure of the couplings $d_{ \pm \sigma}$ in Eq. (2.4). For the transitions in $s, d$, and $g$ channels in case 1 above $d_{+\sigma}=d_{-\sigma}$ by inversion symmetry and $d_{ \pm \sigma}$ is $\sigma$ independent. For case 2, we have $d_{+\sigma}=-d_{-\sigma}$ and also $\sigma$ independent, although the fermions now couple to a projection of the two component order parameter $\vec{\phi} \cdot \vec{d}$, while the bosonic gradient term generally involves both components of the order parameter. The spin-liquid case also has $d_{+\sigma}=-d_{-\sigma}$ and $\sigma$ independent, and $\phi$ is associated with the transverse component of the spatial gauge field in the Coulomb gauge; $;^{37-40}$ moreover the spin liquid has $r=0$ by gauge invariance. Finally, the Ising ferromagnet case 3 has $d_{+\sigma}=d_{-\sigma}$ and $d_{ \pm \uparrow}=$ $-d_{ \pm 1}$.

We note that for transitions in nonzero angular momentum channels, the coupling $d$ vanishes along certain axes in the Brillouin zone. The intersections of these axes with the Fermi surface are known as cold spots, as the fermion coupling to the order parameter at these points involves additional derivatives and is much weaker. The scaling theory that follows only describes the Fermi surface away from cold spots.

It is convenient to rescale coordinates and fields in Eq. (2.4), $x=\left(2 m v_{F}\right)^{-1} \tilde{x}, \psi=v_{F}^{-1 / 2} \widetilde{\psi}, \phi=\frac{1}{2 m \mid d]} \widetilde{\phi}$. We drop the tildes in what follows. Then,

$$
\begin{aligned}
L= & \psi_{+\sigma}^{\dagger}\left(\eta \partial_{\tau}-i \partial_{x}-\partial_{y}^{2}\right) \psi_{+\sigma}+\psi_{-\sigma}^{\dagger}\left(\eta \partial_{\tau}+i \partial_{x}-\partial_{y}^{2}\right) \psi_{-\sigma} \\
& -\lambda_{+\sigma} \phi \psi_{+\sigma}^{\dagger} \psi_{+\sigma}-\lambda_{-\sigma} \phi \psi_{-\sigma}^{\dagger} \psi_{-\sigma}+\frac{1}{2 e^{2}}\left(\partial_{y} \phi\right)^{2}+\frac{r}{2} \phi^{2}
\end{aligned}
$$

with $e^{2}=2 m d^{2} / v_{F}, r=r_{0} /\left(2 m d^{2}\right), \eta=2 m$, and $\lambda_{s \sigma}=d_{s \sigma} /|d|$, and we will henceforth drop the subscript $k_{0}$ on $L$. We note that as usual, the relation between the parameters of the effective theory and the original model should not be taken literally. Rather, in the critical regime, we have $r_{0}-r_{0 c}$ $=Z_{r}\left(r-r_{c}\right)$, where $r_{c}$ and $r_{0 c}$ denote the critical points of the effective theory and the microscopic theory, respectively. Moreover, the original fields and the fields defined in each patch of the Fermi surface are related by

$$
\begin{aligned}
& \phi(\vec{q}, \omega) \sim Z_{\phi}^{1 / 2} K \phi_{\text {patch }}\left(K q_{x}, q_{y}, \omega\right), \\
& \psi(\vec{q}, \omega) \sim Z_{\psi}^{1 / 2} K \psi_{\text {patch }}\left(K q_{x}, q_{y}, \omega\right) .
\end{aligned}
$$

Note that the "metric factors" $K, Z_{r}, e^{2}, Z_{\psi}, Z_{\phi}$ are generally dependent on the direction of the boson momentum $\hat{q}$ and the cutoff of the low-energy theory $\Lambda$. 


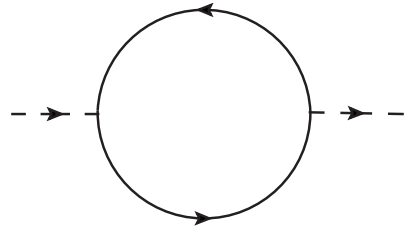

(a)

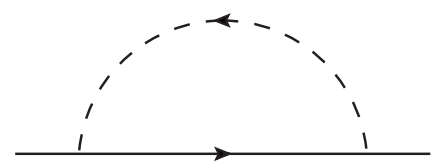

(b)

FIG. 2. One-loop contributions to (a) boson and (b) fermion self-energies.

For brevity, we will only present explicit calculations for the case that does not involve spin (Ising-nematic transition and spin liquid); the extension of the results to the Ising ferromagnet case will be noted. Moreover, we extend the number of spin components (flavors) to $N$ from the physical value $N=2$ with the view towards performing a large- $N$ expansion. For this purpose, it is convenient to rescale $e^{2}$ and $r$, yielding our Lagrangian in its final form

$$
\begin{aligned}
L= & \sum_{s= \pm} \psi_{s}^{\dagger}\left(\eta \partial_{\tau}-i s \partial_{x}-\partial_{y}^{2}\right) \psi_{s}-\sum_{s= \pm} \lambda_{s} \phi \psi_{s}^{\dagger} \psi_{s} \\
& +\frac{N}{2 e^{2}}\left(\partial_{y} \phi\right)^{2}+\frac{N r}{2} \phi^{2} .
\end{aligned}
$$

Here and below we suppress the flavor index. To reiterate, $\left|\lambda_{s}\right|=1$, and the Ising-nematic case has $\lambda_{+}=\lambda_{-}$and the spinliquid case [i.e., Fermi surface coupled to a U(1) gauge field] has $\lambda_{+}=-\lambda_{-}$.

\section{ONE-LOOP PROPAGATORS}

To gain some insight into the low-energy properties of the theory, Eq. (2.7), it is useful to compute the one-loop boson and fermion self-energies.

The one-loop boson polarization in Fig. 2(a) is given by

$$
\Pi_{0}(q)=N \int \frac{d l_{\tau} d^{2} \vec{l}}{(2 \pi)^{3}} G_{s}^{0}(l) G_{s}^{0}(l+q) .
$$

We first evaluate this diagram with a bare fermion propagator,

$$
G_{s}^{0}(k)=\frac{1}{-i \eta k_{\tau}+s k_{x}+k_{y}^{2}} .
$$

The resulting polarization function takes on a characteristic Landau-damped form,

$$
\begin{aligned}
\Pi_{0}(q) & =N \int \frac{d l_{\tau} d l_{y}}{(2 \pi)^{2}} \frac{i\left[\theta\left(l_{\tau}\right)-\theta\left(l_{\tau}+q_{\tau}\right)\right]}{-i \eta q_{\tau}+2 q_{y} l_{y}+q_{x}+q_{y}^{2}}+(\vec{q} \rightarrow-\vec{q}) \\
& =\frac{N q_{\tau}}{2 \pi} \int \frac{d l_{y}}{2 \pi} \frac{(-i)}{-i \eta q_{\tau}+2 q_{y} l_{y}+q_{x}+q_{y}^{2}}+(\vec{q} \rightarrow-\vec{q}) \\
& =c_{b} N \frac{\left|q_{\tau}\right|}{\left|q_{y}\right|}, \quad c_{b}=\frac{1}{4 \pi} .
\end{aligned}
$$

Note that $\eta$ has dropped out of the final result. We are interested above only in the singular contribution to $\Pi_{0}$ and this is insensitive to orders of integration: so unlike the conventional order, we have integrated over $l_{x}$ before $l_{\tau}$. We include the random-phase approximation (RPA) polarization bubble, Eq. (3.3), into the bosonic propagator $D(q)$ to obtain

$$
D(q)=\frac{1}{N}\left(c_{b} \frac{\left|q_{\tau}\right|}{\left|q_{y}\right|}+\frac{q_{y}^{2}}{e^{2}}+r\right)^{-1} .
$$

Note that the $q_{y}^{2}$ term is not renormalized by the polarization contribution at this order and the bare coefficient represents the phenomenological contribution of higher-energy modes.

The one-loop correction to the fermion propagator is given by Fig. 2(b). For simplicity, we work at the critical point and set $r=0$. Then, the fermion self-energy assumes a non-Fermi-liquid form

$$
\begin{aligned}
\Sigma_{s}(k) & =-\int \frac{d l_{\tau} d^{2} \vec{l}}{(2 \pi)^{3}} D(l) G_{s}^{0}(k-l) \\
& =-\frac{i}{2 N} \int \frac{d l_{\tau} d l_{y}}{(2 \pi)^{2}}\left(c_{b} \frac{\left|l_{\tau}\right|}{\left|l_{y}\right|}+\frac{l_{y}^{2}}{e^{2}}\right)^{-1} \times \operatorname{sgn}\left(k_{\tau}-l_{\tau}\right) \\
& =-\frac{i c_{f}}{N} \operatorname{sgn}\left(k_{\tau}\right)\left|k_{\tau}\right|^{2 / 3}, \quad c_{f}=\frac{2}{\sqrt{3}}\left(\frac{e^{2}}{4 \pi}\right)^{2 / 3} .
\end{aligned}
$$

Note, again, that $\eta$ has dropped out of the result. Incorporating this correction into the fermion propagator,

$$
G_{s}(k)=\left[-\frac{i c_{f}}{N} \operatorname{sgn}\left(k_{\tau}\right)\left|k_{\tau}\right|^{2 / 3}+s k_{x}+k_{y}^{2}\right]^{-1} .
$$

Here we have dropped the bare fermion time derivative term proportional to $\eta$, which is irrelevant at low energies compared to the dynamically induced self-energy, Eq. (3.5).

As is well known, ${ }^{37}$ the one-loop expressions (3.3) and (3.5) actually satisfy the Eliashberg-type equations, in which the lines of Fig. 2 become self-consistent propagators. In what follows, we will use these self-consistent propagators, Eqs. (3.4) and (3.6), in our calculations and drop self-energy corrections like those in Fig. 2.

\section{SCALING AND RENORMALIZATION}

As has been argued by a number of authors, ${ }^{37-40}$ a useful starting point for the renormalization-group analysis of the theory, Eq. (2.7), is obtained by using the scaling,

$$
k_{x} \rightarrow s^{2} k_{x}, \quad k_{y} \rightarrow s k_{y}, \quad \omega \rightarrow s^{3} \omega,
$$




$$
\psi(x, y, \tau) \rightarrow s^{2} \psi\left(s^{2} x, s y, s^{3} \tau\right), \quad \phi(x, y, \tau) \rightarrow s^{2} \phi\left(s^{2} x, s y, s^{3} \tau\right) .
$$

This scaling is suggested by the one-loop calculation of fermion and boson propagators in Eqs. (3.4) and (3.6). The bare fermion time derivative term $\psi^{\dagger} \partial_{\tau} \psi$ is irrelevant under this scaling and so we will take the limit $\eta \rightarrow 0^{+}$. Note that neither of the one-loop corrections, Eqs. (3.3) and (3.5), depend upon $\eta$.

Alternatively, note that the scaling of time in Eq. (4.1) could also have been derived by demanding that the "Yukawa coupling" $\lambda_{s}$ be invariant. This avoids the somewhat unnatural appeal to the one-loop self-energy to set bare scaling dimensions and yields all the scaling dimensions in Eq. (4.1) by a simple rescaling of the bare Lagrangian $L$ in Eq. (2.7). Of course, once we have set $\lambda_{s}$ to be invariant, then the coupling $\eta$ becomes irrelevant. These features of the scaling analysis are shared by the theory of the nematic transition in $d$-wave superconductors in Ref. 36.

Note also the different scaling of spatial momenta $k_{x}$ and $k_{y}$ in Eq. (4.1). The main physical consequence of scaling of the momentum $k_{y}$ is the effective decompactification of the Fermi surface, which allows one to focus on a theory with two Fermi patches. Also observe that under, Eq. (4.1), the $\left(\partial_{x} \phi\right)^{2}$ part of the boson tree level action is irrelevant, which justifies omitting this term in Eqs. (2.4) and (2.7).

Apart from the fermion time derivative term and the relevant mass perturbation $\left(r \rightarrow s^{-2} r\right)$, all the terms in Lagrangian (2.7) are marginal. Higher-order perturbations to Eq. (2.7), consistent with the $Z_{2}$ symmetry of the order parameter, such as a $\phi^{4}$ term, are irrelevant.

We would like to note that for the case of the Isingnematic (or $g$-wave) transition the low-energy action, Eq. (2.7), does not possess a $\phi \rightarrow-\phi$ symmetry. This is due to the fact that the direction of bosonic momentum $\vec{q}$ is transformed under $\pi / 2$ rotations (reflections) and hence the physics is controlled by a different pair of patches of the Fermi surface. Hence, in principle, it is possible that in the kinematic regime of interest a $\phi^{3}$ term is generated by the renormalization-group process. Such a term would be marginal under the scaling in Eq. (4.1). A linear term in $\phi$ can also be generated by the effective theory. However, the onepoint function has momentum $\vec{q}=0$ and, hence, does not belong to any particular kinematic regime. In practice, we can demand that the expectation value of $\phi$ is zero in the disordered phase by tuning the coefficient of the $\phi$-linear term. In any case, as we will show below, there exists a Ward identity, which guarantees that if these terms are initially zero, they are not generated by the RG of the low-energy theory, Eq. (2.7). Note that for the case of the spin-liquid or Ising ferromagnet transitions, the low-energy theory, Eq. (2.7), respects the time-reversal symmetry which maps Fermi patches at $k_{0}$ and $-k_{0}$ into each other and, hence, terms odd in $\phi$ are prohibited.

An important observation is that the theory, Eq. (2.7), lacks an expansion parameter. To see this, note that due to the rescaling performed in Sec. II, the engineering dimensions, $\left[k_{x}\right]=\left[k_{y}\right]^{2}$, but the dimension of $\omega$ is kept independent. Then, the coupling constant $e^{2}$ has the dimensions $\left[k_{y}\right]^{3} /[\omega]$. Therefore, $e^{2}$ is a dimensionful quantity and cannot be used as an expansion parameter. Moreover, $e^{2}$ is actually the only parameter in the theory relating frequencies and momenta. Hence, its flow under RG is equivalent to an appearance of a nontrivial dynamical critical exponent.

Note that up to this point we have dropped an allowed relevant fermion chemical potential term,

$$
\Delta L=-\delta \psi_{s}^{\dagger} \psi_{s} .
$$

This term can be absorbed into the definition of the momentum $\vec{k}_{0}$ about which the theory is expanded and, thus, is redundant (note, the scaling dimension $[\delta]=\left[k_{x}\right]=2$ ). Nevertheless, it is convenient to leave this term in the Lagrangian for renormalization-group purposes. We assume that when the theory is tuned to the criticality $r=r_{c}$ and the coefficient $\delta$ is set to $\delta=\delta_{c}$, the Fermi surface passes through the points $\vec{k}_{0},-\vec{k}_{0}$.

We now discuss the renormalization of our theory. The Lagrangian contains four marginal operators, which each requires a renormalization constant. However, as we will argue below, emergent low-energy symmetries of the theory imply certain relations between these constants. Moreover, the two relevant operators, have the same bare dimension, $[r]=[\delta]$ $=2$. Thus, we need to consider possible mixing between these operators.

\section{A. Rotational symmetry}

Observe that the initial shape of the Fermi surface does not enter the low-energy theory, Eq. (2.7). In fact, we could have started with a circular Fermi surface with $k_{F}=m v_{F}$. This is reflected by the fact that Eq. (2.7) has an emergent continuous "rotational symmetry,"

$$
\begin{aligned}
\phi(x, y) & \rightarrow \phi(x, y+\theta x), \\
\psi_{s}(x, y) & \rightarrow e^{-i s\left[(\theta / 2) y+\left(\theta^{2} / 4\right) x\right]} \psi_{s}(x, y+\theta x) .
\end{aligned}
$$

Equivalently in momentum space,

$$
\begin{aligned}
\phi\left(q_{x}, q_{y}\right) & \rightarrow \phi\left(q_{x}-\theta q_{y}, q_{y}\right), \\
\psi_{s}\left(q_{x}, q_{y}\right) & \rightarrow \psi_{s}\left(q_{x}-\theta q_{y}-s \frac{\theta^{2}}{4}, q_{y}+s \frac{\theta}{2}\right) .
\end{aligned}
$$

Note that the rotation angle $\theta$ becomes noncompact and the rotation group becomes $\mathbb{R}$ instead of $\mathrm{U}(1)$. This is a consequence of the effective decompactification of the Fermi surface. Moreover, due to the anisotropic scaling $\theta$ is now dimensionful $[\theta]=\left[k_{y}\right]$. In fact, the situation is analogous to the transformation of the Lorentz symmetry to Galilean invariance in the nonrelativistic limit $\omega \ll c|\vec{q}|$. Here the role of $\omega$ is played by $q_{x}$ and the role of $|\vec{q}|$ by $q_{y}$.

The symmetry in Eq. (4.4) implies the following form of the bosonic and fermionic Green's functions (we suppress the frequency dependence),

$$
D\left(q_{x}, q_{y}\right)=D\left(q_{y}\right),
$$




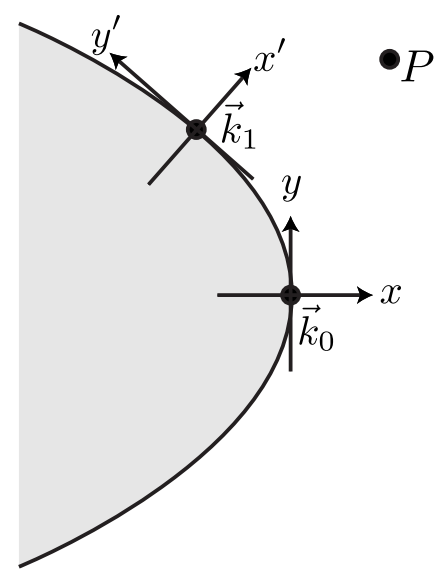

FIG. 3. The momentum of the fermion at point $P$ can be measured with respect to either the coordinate system at $\vec{k}_{0}$ or that at $\vec{k}_{1}$.

$$
G_{s}\left(q_{x}, q_{y}\right)=G\left(s q_{x}+q_{y}^{2}\right) .
$$

In particular, the form of the fermionic Green's function implies that the terms $\psi_{s}^{\dagger}\left(-i s \partial_{x}\right) \psi_{s}$ and $\psi_{s}\left(-\partial_{y}^{2}\right) \psi_{s}$ in the Lagrangian (2.7) must renormalize in the same way. Physically, this means that the curvature radius of the Fermi surface $K$ does not flow under RG (i.e., $K$ has a limit as the cutoff $\Lambda$ $\rightarrow 0$ ).

The identities (4.5) and (4.6) ensure that the Green's function at a given physical momentum remain invariant under small changes in the choice of the points $\pm \vec{k}_{0}$ on the Fermi surface about which the field theory is defined. Let us demonstrate this explicitly using Fig. 3. We set the coordinate system so that $\vec{k}_{0}=(0,0)$ and measure the momentum of a fermion at the point $P$ to be $\left(q_{x}, q_{y}\right)$. Now let us shift to the field theory defined at the Fermi-surface point $\vec{k}_{1}=\left(\kappa_{x}, \kappa_{y}\right)$. As this point has to be on the Fermi surface, we have $\kappa_{x}$ $+\kappa_{y}^{2}=0$. We denote the coordinates of the point $P$ in the new coordinate system by $\left(q_{x}^{\prime}, q_{y}^{\prime}\right)$. These are obtained from the old coordinates by a shift in origin followed by a rotation by an angle $\theta$, where $\tan \theta=2 \kappa_{y}$; this yields

$$
\begin{gathered}
q_{x}^{\prime}=q_{x}-\kappa_{x}+2 \kappa_{y}\left(q_{y}-\kappa_{y}\right), \\
q_{y}^{\prime}=q_{y}-\kappa_{y},
\end{gathered}
$$

where we only keep terms to the needed accuracy of $\mathcal{O}\left(x, y^{2}\right)$. It can now be verified that $q_{x}^{\prime}+q_{y}^{\prime 2}=q_{x}+q_{y}^{2}$, and so by Eq. (4.6) the fermion Green's function remains invariant under the change in the Fermi-surface reference point. Also, by choosing $\kappa_{y}=q_{y}$ we can set $q_{y}^{\prime}=0$ and then $q_{x}+q_{y}^{2}$ is identified as the invariant measuring the distance between $P$ and the closest point on the Fermi surface. For the boson Green's function, there is no shift in origin of the coordinates, and the corresponding transformation is $q_{x}^{\prime}=q_{x}+2 \kappa_{y} q_{y}, q_{y}^{\prime}=q_{y}$, leading to Eq. (4.5).

These invariances are essential in ensuring the consistency of our description of each pair of time-reversed Fermisurface points by a separate 2+1-dimensional field theory. Note that such a consistency requirement would not have arisen if we had used a $1+1$ dimensional field theory at each
Fermi-surface point ${ }^{27,28,41-45}$ because then every fermion momentum would appear only in the theory defined at the closest point on the Fermi surface. In our case, we are free to use the 2+1-dimensional theory at this closest point or at any of the neighboring points. Before concluding this section, we would like to point out that in the case of the Ising-nematic transition, the "rotational symmetry," Eq. (4.4), is not related in any way to "large" rotations by $\pi / 2$, which are actually not implemented in the low-energy theory.

\section{B. Ward identities}

We now examine the consequences of Ward identities associated with the global symmetries of Eq. (2.7). Similar consequences were implicit in the analysis of the superconducting case in Ref. 36. Here we will present a more formal analysis, which also shows that Eq. (3.20) in Ref. 36 holds to all orders in $1 / N$.

The low-energy theory, Eq. (2.7), has two continuous global U(1) symmetries. The first of these is related to the conservation of particle number,

$$
\mathrm{U}(1)_{F}: \quad \psi_{+} \rightarrow e^{i \alpha} \psi_{+}, \quad \psi_{-} \rightarrow e^{i \alpha} \psi_{-} .
$$

The conserved current associated with this symmetry is,

$$
\begin{aligned}
\left(j_{\tau}, j_{x}, j_{y}\right)_{F}= & {\left[i \eta\left(\psi_{+}^{\dagger} \psi_{+}+\psi_{-}^{\dagger} \psi_{-}\right), \psi_{+}^{\dagger} \psi_{+}-\psi_{-}^{\dagger} \psi_{-},\right.} \\
& \left.-i\left(\psi_{+}^{\dagger} \stackrel{\leftrightarrow}{\partial}_{y} \psi_{+}+\psi_{-}^{\dagger} \stackrel{\leftrightarrow}{\partial}_{y} \psi_{-}\right)\right] .
\end{aligned}
$$

For the spin-liquid problem, the gauge field $\phi$ couples precisely to the $x$ component of $j_{F}$.

The second U(1) symmetry is lattice translation. Indeed, $\psi_{+}$and $\psi_{-}$come from opposite points in the Brillouin zone and, hence, transform under general lattice translations as

$$
\mathrm{U}(1)_{T}: \quad \psi_{+} \rightarrow e^{i \alpha} \psi_{+}, \quad \psi_{-} \rightarrow e^{-i \alpha} \psi_{-} .
$$

The conserved current associated with this symmetry is

$$
\begin{aligned}
\left(j_{\tau}, j_{x}, j_{y}\right)_{T}= & {\left[i \eta\left(\psi_{+}^{\dagger} \psi_{+}-\psi_{-}^{\dagger} \psi_{-}\right), \psi_{+}^{\dagger} \psi_{+}+\psi_{-}^{\dagger} \psi_{-},\right.} \\
& \left.-i\left(\psi_{+}^{\dagger} \stackrel{\leftrightarrow}{\partial}_{y} \psi_{+}-\psi_{-}^{\dagger} \stackrel{\leftrightarrow}{\partial}_{y} \psi_{-}\right)\right] .
\end{aligned}
$$

Observe that the Ising-nematic order parameter $\phi$ couples to the $x$ component of $j_{T}$. Note that despite the similarity of the spin-liquid and Ising-nematic problems, there is an important difference. In the spin-liquid case, the gauge field couples to the fermion current on all energy scales. In the case of the Ising-nematic transition, the order parameter couples to a conserved current only at low energies.

We note in passing that for an Ising ferromagnet transition, the current to which the order parameter couples is related to the symmetry,

$$
\begin{gathered}
\mathrm{U}(1)_{I}: \quad \psi_{+\uparrow} \rightarrow e^{i \alpha} \psi_{+}, \quad \psi_{-\uparrow} \rightarrow e^{-i \alpha} \psi_{-}, \\
\psi_{+\downarrow} \rightarrow e^{-i \alpha} \psi_{+\downarrow}, \quad \psi_{-\downarrow} \rightarrow e^{i \alpha} \psi_{-\downarrow} .
\end{gathered}
$$

In fact, this is not a symmetry of the underlying theory but only of the low-energy Lagrangian (2.4). The symmetry is broken by four-Fermi interactions, which are however irrelevant under the scaling in Eq. (4.1).

Current conservation implies that the insertion of $\partial_{\tau} j_{\tau}$ $+\partial_{x} j_{x}+\partial_{y} j_{y}$ into any correlation function is zero, up to con- 
tact terms (we have dropped the current subscript; the current, which couples to the order parameter is implicitly assumed). We note that the temporal component of the currents, Eqs. (4.9) and (4.11), has a coefficient $\eta$ in front and, therefore, can be set to zero in the kinematic regime of interest. We, thus, have $\partial_{x} j_{x}+\partial_{y} j_{y} \sim 0$. Defining the oneparticle irreducible polarization function,

$$
\Pi_{i j}(q)=\int d \tau d^{2} x e^{i q_{\tau} \tau-i \vec{q} \cdot \vec{x}}\left\langle j_{i}(x) j_{j}(0)\right\rangle_{1 P I},
$$

we have

$$
q_{x} \Pi_{x x}(q)+q_{y} \Pi_{y x}(q)=0 .
$$

We note that $\Pi_{x x}(q)=\Pi_{x x}\left(q_{\tau}, q_{y}\right)$ is precisely the irreducible boson self-energy. Hence,

$$
\Pi_{y x}\left(q_{\tau}, q_{x}, q_{y}\right)=-\frac{q_{x}}{q_{y}} \Pi_{x x}\left(q_{\tau}, q_{y}\right) .
$$

Power counting indicates that $\Pi_{x x}$ has the following UV structure:

$$
\Pi_{x x}\left(q_{\tau}, q_{y}\right)=K_{1}+K_{2} r+K_{3} q_{y}^{2},
$$

where $K_{1} \sim \Lambda^{2}, K_{2}, K_{3} \sim \log \Lambda$, and $\Lambda$ is the UV cutoff with dimensions of $q_{y}$. For $\Pi_{y x}\left(q_{\tau}, q_{x}, q_{y}\right)$ to have an analytic UV behavior (as again expected from power counting), we must have

$$
K_{1}=K_{2}=0 .
$$

Thus, the coefficient of the mass operator $\phi^{2}$ requires no renormalization (i.e., the metric factor $Z_{r}$ has a limit as $\Lambda$ $\rightarrow 0$ ).

An interesting question is whether the polarization function $\Pi_{x x}$ actually vanishes for $q_{y} \rightarrow 0$ as suggested by Eq. (4.14). However, for finite $q_{\tau}$ we already know from oneloop calculations that such a limit does not exist within the scaling regime as

$$
\Pi_{x x}\left(q_{\tau}, q_{y}\right)_{1 \text { loop }}=c_{b} \frac{\left|q_{\tau}\right|}{\left|q_{y}\right|}, \quad \Pi_{y x}\left(q_{\tau}, q_{x}, q_{y}\right)_{1 \text { loop }}=-c_{b} \frac{q_{x}}{q_{y}} \frac{\left|q_{\tau}\right|}{\left|q_{y}\right|} .
$$

However, one might hope that the limits $\lim _{q_{y} \rightarrow 0} \lim _{q_{\tau} \rightarrow 0} \Pi_{x x}\left(q_{\tau}, q_{y}\right), \Pi_{x y}\left(q_{\tau}, q_{x}, q_{y}\right)$ do exist. In this case, we would conclude

$$
\lim _{q_{y} \rightarrow 0} \lim _{q_{\tau} \rightarrow 0} \Pi_{x x}\left(q_{\tau}, q_{y}\right)=0,
$$

which would be a stronger statement than the nonrenormalization of the mass term. Otherwise, if the limit above exists only for $\Pi_{x x}$ by not $\Pi_{x y}$ then,

$$
\lim _{q_{y} \rightarrow 0} \lim _{q_{\tau} \rightarrow 0} \Pi_{x x}\left(q_{\tau}, q_{y}\right)=c_{r} r
$$

with $c_{r}$-some universal constant. We have explicitly checked that to three-loop order $c_{r}=0$ and the strong form of the nonrenormalization identity, Eq. (4.16), holds.

One can generalize the discussion above to higher-order correlation functions of the order parameter. Ward identities imply that the effective potential for the $\phi$ field is not renormalized from its tree-level form,

$$
V(\phi)=\frac{r}{2} \phi^{2}
$$

This property is also shared by the theory of the nematic transition in a $d$-wave superconductor. ${ }^{35,36}$ In particular, no $\phi^{3}$ term is induced in the Lagrangian by the renormalizationgroup process if this term is originally zero. (Note that if a $\phi^{3}$ term is initially present, correlation functions of currents no longer coincide with the correlation functions of the order parameter, and the Ward identities do not constrain the renormalization properties of the theory). The effective potential, Eq. (4.18), becomes unstable for $r<0$. Thus, we expect that in the ordered phase the theory is controlled by dangerously irrelevant operators, such as $\phi^{4}$.

Finally, one can derive a Ward identity for the fermion boson vertex,

$$
q_{x} \Gamma_{x}(q, p, p+q)+q_{y} \Gamma_{y}(q, p, p+q)=G^{-1}(p+q)-G^{-1}(p)
$$

with

$$
\begin{aligned}
\Gamma_{i}(q, p, p+q)= & \int d x_{\tau} d^{2} x d y_{\tau} d^{2} y e^{-i q_{\tau} x_{\tau}+i \vec{q} \cdot \vec{x}} e^{i(p+q)_{\tau} y_{\tau}-i(\vec{p}+\vec{q}) \cdot \vec{y}} \\
& \times\left\langle j_{i}(x) \psi(y) \psi^{\dagger}(0)\right\rangle_{1 P I} \\
G(p)= & \int d \tau d^{2} x e^{i q_{\tau} \tau-i \vec{q} \cdot \vec{x}}\left\langle\psi(x) \psi^{\dagger}(0)\right\rangle
\end{aligned}
$$

$\Gamma_{x}$ is precisely the irreducible fermion-boson vertex. Power counting gives UV structure of $\Gamma_{x}$ and $G^{-1}$

$$
\begin{gathered}
\Gamma_{x}(q, p, p+q)=C_{1}, \\
G^{-1}(p)=C_{2}+C_{3}\left(p_{x}+p_{y}^{2}\right) .
\end{gathered}
$$

Thus, for the UV behavior of $\Gamma_{y}$ to be analytic in external momenta, $C_{1}=C_{3}$. Therefore, the vertex and the fermion selfenergy renormalize in the same way. Hence, the boson field requires no field-strength renormalization (i.e., the metric factor $Z_{\phi}$ has a limit as $\Lambda \rightarrow 0$ ).

Before concluding this section, we would like to note that perturbation theory based on self-consistent propagators, Eqs. (3.4) and (3.6), actually does not respect the Ward identities. This is due to the fact that these one-loop propagators include the fermion self-energy correction but not the vertex correction. However, since the fermion self-energy is only frequency dependent, Ward identities involving currents at zero external frequency are still respected.

\section{RG equations}

From the discussion above, we conclude that at criticality, our theory needs only two renormalizations: a rescaling of the field strength of the fermion field $\psi$ and a renormalization of $e^{2}$,

$$
\psi=Z_{\psi}^{1 / 2} \psi_{r}, \quad e^{2}=Z_{e} e_{r}^{2}
$$

Here the subscript $r$ denotes renormalized quantities and we define renormalized irreducible correlation functions of $n_{b}$ boson and $n_{f}$ fermion fields as 


$$
\Gamma_{r}^{n_{b}, n_{f}}=Z_{\psi}^{n_{f} / 2} \Gamma^{n_{b}, n_{f}} .
$$

Both $Z_{\psi}$ and $Z_{e}$ are functions of $\Lambda / \mu$, where $\mu$ is a renormalization scale (which we choose to have dimensions of $q_{y}$ ), and of the number of fermion flavors $N$. As $e^{2}$ is dimensionful, $Z_{\psi}$ and $Z_{e}$ cannot depend on it. We introduce the anomalous dimensions,

$$
\begin{gathered}
b=\Lambda \frac{\partial}{\partial \Lambda} \log Z_{e}, \\
\eta_{\psi}=-\Lambda \frac{\partial}{\partial \Lambda} \log Z_{\psi} .
\end{gathered}
$$

The constants $\eta_{\psi}$ and $b$ are expected to be pure universal numbers, independent of $\Lambda / \mu$.

Away from criticality, we recall that by the Ward identity, the coupling $r$ does not renormalize. On the other hand, the coupling $\delta$ can pick up a renormalization linear in $r$,

$$
\delta=\delta_{c}+\delta_{r}+Z_{r \delta} e_{r}^{2} r
$$

with $Z_{r \delta}$ again a function of $\Lambda / \mu$ only. In what follows, we denote $\delta-\delta_{c}$ as $\delta$ for brevity. Note that there is no renormalization constant in front of $\delta_{r}$ since a finite change in $\delta$ only shifts the value of $k_{x}$ in correlation functions,

$$
\Gamma^{n_{b}, n_{f}}(\{p\}, \delta+a)=\Gamma^{n_{b}, n_{f}}(\{p-s a \hat{x}\}, \delta),
$$

where $s= \pm 1$ for momenta of fermions $\psi_{ \pm}$and $s=0$ for boson momenta. We let

$$
\alpha=Z_{e}^{-1} \Lambda \frac{\partial}{\partial \Lambda} Z_{r \delta}
$$

Now, differentiating Eq. (4.25) we obtain the renormalization-group equations,

$$
\begin{aligned}
& \left(\Lambda \frac{\partial}{\partial \Lambda}+b e^{2} \frac{\partial}{\partial e^{2}}+\alpha e^{2} r \frac{\partial}{\partial \delta}-\frac{n_{f}}{2} \eta_{\psi}\right) \\
& \times \Gamma^{n_{b}, n_{f}}\left(\left\{p_{y}\right\},\left\{p_{x}\right\},\{\omega\}, r, \delta, e^{2}, \Lambda\right)=0 .
\end{aligned}
$$

It is convenient to get rid of the derivative with respect to $\delta$ in Eq. (4.31). To do so, let the location of the Fermi surface of fermion $\psi_{+}$at finite $\delta$ and $r$ be given by $k_{x}+k_{y}^{2}$ $=\Delta k\left(r, \delta, e^{2}, \Lambda\right)$. Then, $\Delta k$ is clearly a physical quantity and must satisfy

$$
\left(\Lambda \frac{\partial}{\partial \Lambda}+b e^{2} \frac{\partial}{\partial e^{2}}+\alpha e^{2} r \frac{\partial}{\partial \delta}\right) \Delta k\left(r, \delta, e^{2}, \Lambda\right)=0 .
$$

We will solve this equation shortly. However, first note that

$$
\frac{\partial \Delta k}{\partial \delta}=1
$$

Now, it is convenient to expand momenta around the physical Fermi surface, defining

$$
\widetilde{\Gamma}^{n_{b}, n_{f}}\left(\{p\}, r, \delta, e^{2}, \Lambda\right)=\Gamma^{n_{b}, n_{f}}\left(\left\{p+s \Delta k\left(r, \delta, e^{2}, \Lambda\right) \hat{x}\right\}, r, \delta, e^{2}, \Lambda\right) .
$$

The resulting $\widetilde{\Gamma}$ is independent of $\delta$ and by Eqs. (4.29) and (4.31)-(4.33) satisfies,

$$
\left(\Lambda \frac{\partial}{\partial \Lambda}+b e^{2} \frac{\partial}{\partial e^{2}}-\frac{n_{f}}{2} \eta_{\psi}\right) \widetilde{\Gamma}^{n_{b}, n_{f}}\left(\left\{p_{y}\right\},\left\{p_{x}\right\},\{\omega\}, r, e^{2}, \Lambda\right)=0
$$

By dimensional analysis,

$$
\widetilde{\Gamma}^{n_{b}, n_{f}}=\Lambda^{6-2 n_{f}-2 n_{b}}\left(e^{2}\right)^{n_{f} / 2-1} f^{n_{b}, n_{f}}\left(\left\{\frac{p_{y}}{\Lambda}\right\},\left\{\frac{p_{x}}{\Lambda^{2}}\right\},\left\{\frac{\omega e^{2}}{\Lambda^{3}}\right\}, \frac{\Lambda^{2} r}{\mu^{2}}\right)
$$

and solving the RG equation, we obtain

$$
\begin{aligned}
f^{n_{b}}, n_{f} & \left(s\left\{\tilde{p}_{y}\right\}, s^{2}\left\{\tilde{p}_{x}\right\}, s^{3-b}\{\widetilde{\omega}\}, s^{2-b} \widetilde{r}\right) \\
& =s^{6-b+\left(b-\eta_{\psi^{-}}-4\right) n_{f} / 2-2 n_{b}} f^{n_{b}, n_{f}}\left(\left\{\widetilde{p}_{y}\right\},\left\{\widetilde{p}_{x}\right\},\{\widetilde{\omega}\}, \widetilde{r}\right) .
\end{aligned}
$$

Hence, the critical theory is invariant under

$$
p_{y} \rightarrow s p_{y}, \quad p_{x} \rightarrow s^{2} p_{x}, \quad \omega \rightarrow s^{z} \omega
$$

with

$$
z=3-b
$$

where $z$ is the dynamic critical exponent. Note that we have defined $z$ with reference to length scales associated with the direction tangent to the Fermi surface $(y)$; as indicated in Eq. (4.38), length scales orthogonal to the Fermi-surface scale as the square of length scales tangent to the Fermi surface. Moreover, if we define $\xi$ as the correlation length along the $y$ direction then upon approaching the critical point, $\xi \sim r^{-\nu}$, with

$$
\nu=\frac{1}{z-1} .
$$

Note that by combining Eqs. (4.24), (4.26), and (4.39) we can write down the RG equation for the coupling $e$,

$$
\left.\Lambda \frac{\partial e^{2}}{\partial \Lambda}\right|_{e_{r}^{2}, \mu}=-(z-3) e^{2}
$$

This shows that the renormalization of the coupling $e$ is directly related to the dynamic critical exponent, as we had claimed earlier.

Now, let us consider a few explicit examples of correlation functions. For the bosonic two-point function we have

$$
D^{-1}\left(q_{y}, \omega\right)=\operatorname{rg}\left[q_{y}\left(r e^{2} \Lambda^{z-3}\right)^{-1 /(z-1)}, \omega\left(r^{z} e^{2} \Lambda^{z-3}\right)^{-1 /(z-1)}\right] .
$$

Note that,

$$
\lim _{q_{y} \rightarrow 0} \lim _{\omega \rightarrow 0} D^{-1}\left(q_{y}, \omega\right)=\operatorname{rg}(0,0),
$$

i.e., the Ising-nematic susceptibility satisfies $\chi \sim r^{-\gamma}$ with the exponent

$$
\gamma=1
$$

We may also write more succinctly,

$$
D^{-1}\left(q_{y}, \omega\right) \propto \xi^{-(z-1)} g\left(q_{y} \xi, \omega e^{2} \Lambda^{z-3} \xi^{z}\right) .
$$


So far, we have been concentrating on a fixed direction of bosonic momentum $\vec{q}$. Now let us study the dependence of the result on $\hat{q}$. Using Eq. (2.6),

$$
\begin{aligned}
& D^{-1}(\vec{q}, \omega) \\
& =Z_{\phi}^{-1} K^{-1} Z_{r}^{-1} r_{0} \\
& \quad \times g\left[|\vec{q}|\left(Z_{r}^{-1} e^{2} \Lambda^{z-3} r_{0}\right)^{-1 /(z-1)}, \omega\left(Z_{r}^{-z} e^{2} \Lambda^{z-3} r_{0}^{z}\right)^{-1 /(z-1)}\right],
\end{aligned}
$$

where for brevity $r_{0}$ is taken to denote the deviation from the critical point. We concentrate on the static limit $\omega=0$. In a Fermi liquid, the susceptibility must have a continuous limit as $\vec{q} \rightarrow 0$. Therefore, we conclude that the combination $Z_{\phi} K Z_{r}$ must be independent of the direction $\hat{q}$. This is quite plausible as neither of the constants run under RG.

Now let us look at the behavior of susceptibility at the critical point,

$$
D^{-1}\left(q_{y}, \omega\right)=\frac{q_{y}^{z-1}}{e^{2} \Lambda^{z-3}} h\left(\frac{\omega e^{2} \Lambda^{z-3}}{q_{y}^{z}}\right) .
$$

In particular, the static susceptibility satisfies

$$
D^{-1}(\vec{q}, 0) \sim a(\hat{q})|\vec{q}|^{z-1} .
$$

In the context of the spin-liquid problem, many studies $^{50,52-56}$ examined the structure of the higher-loop corrections to the susceptibility. In particular, Kim et al. ${ }^{50}$ examined two-loop corrections to $\operatorname{Im} D^{-1}(\vec{q}, \omega)$ for real frequencies $|\omega| \ll|\vec{q}|$, and found no corrections to the leading answer $\sim \omega /\left|q_{y}\right|$ in Eq. (3.4); Fermi-liquid arguments were made $50,52-54,56$ that this functional form held at higher orders. However, this result by itself does not fix the value of $z$; indeed, $\operatorname{Im} D^{-1}(\vec{q}, \omega) \sim \omega /\left|q_{y}\right|$ is consistent with the scaling form, Eq. (4.47), for any $z$. These studies also implicitly assumed a Fermi-liquid picture with $D^{-1}(\vec{q}, \omega=0) \sim \vec{q}^{2}$ and this does imply $z=3$. We will examine $D^{-1}(\vec{q}, \omega=0)$ up to three loops in Sec. V A and find no correction to $z=3$.

Proceeding to the fermion Green's function,

$$
\begin{aligned}
G_{s}^{-1}(\vec{k}, \omega)= & \Lambda^{2}\left(\frac{r e^{2}}{\Lambda^{2}}\right)^{\left(2-\eta_{\psi}\right) /(z-1)} \\
& \times L\left(k\left(r e^{2} \Lambda^{z-3}\right)^{-2 /(z-1)}, \omega\left(r^{z} e^{2} \Lambda^{z-3}\right)^{-1 /(z-1)}\right)
\end{aligned}
$$

with $k=s k_{x}+k_{y}^{2}$ - the distance to the Fermi surface. More compactly

$$
G^{-1}(\vec{k}, \omega) \propto \xi^{-\left(2-\eta_{\psi}\right)} L\left(k \xi^{2}, \omega e^{2} \Lambda^{z-3} \xi^{z}\right) .
$$

A crucial property of the theory that is manifested by the above expression is that the "fermionic correlation length" scales as the square of the "bosonic correlation length."

For $\omega \ll \xi^{-z}, k \ll \xi^{-2}$ we expect the fermion Green's function to assume a Fermi-liquid form,

$$
G(\vec{k}, \omega)=\frac{Z}{-i \omega+v_{F} k} .
$$

By matching to the scaling form,

$$
v_{F} \sim \xi^{-(z-2)}, \quad Z \sim \xi^{\left(z+\eta_{\psi^{-2}}\right)} .
$$

Notice that both the Fermi velocity $v_{F}$ and the residue $Z$ tend to zero as we approach the critical point, albeit with different power laws. Finally, at the quantum critical point,

$$
G^{-1}(\vec{k}, \omega)=\Lambda^{\eta_{\psi}} k^{1-\eta_{\psi} / 2} P\left(\frac{\omega e^{2} \Lambda^{z-3}}{k^{z / 2}}\right),
$$

where we reiterate that $k=s k_{x}+k_{y}^{2}$ is the distance to the Fermi surface. In particular, the self-energy on the Fermi surface scales as

$$
G^{-1}(0, \omega) \sim \omega^{\left(2-\eta_{\psi}\right) / z}
$$

and the static self-energy,

$$
G^{-1}(\vec{k}, 0) \sim k^{1-\eta_{\psi} / 2} .
$$

Moreover, from Eq. (4.53) we can obtain the tunneling density of states,

$$
N(\omega)=\int \frac{d^{2} k}{(2 \pi)^{2}} A(\vec{k}, \omega),
$$

where

$$
A(\vec{k}, \omega)=-\frac{1}{\pi} \operatorname{Im} G\left(\vec{k}, i \omega \rightarrow \omega+i 0^{+}\right) .
$$

The $\vec{k}$ integral in Eq. (4.56) factorizes into integrals over components along and perpendicular to the Fermi surface. The former gives a factor proportional to the perimeter of the Fermi surface while the later yields the frequency dependence,

$$
N(\omega) \sim \omega^{\eta_{\psi} / z}
$$

We remind the reader that the expression in Eq. (4.58) corresponds to the physically observable electron tunneling density of states only in the case of a nematic transition, as for the spin/charge-liquid problem, the physical electron operator is a product of $\psi$ and a boson operator.

Related scaling forms for the fermion Green's function were discussed on a phenomenological basis by Senthil. ${ }^{58}$ However his definition of $z$ differs from ours. We define it using the fermion momentum parallel to the Fermi surface because this is the natural momentum scale appearing also in the boson correlations. He defines it by the fermion momentum orthogonal to the Fermi surface, which scales as the square of the parallel momentum.

Finally, let us discuss the shift of the Fermi surface $\Delta k$. Using Eq. (4.33) in the RG Eq. (4.32), we obtain,

$$
\Delta k=\frac{\alpha}{z-3} r e^{2}+C_{k}\left(r e^{2} \Lambda^{z-3}\right)^{2 v}+\delta .
$$

Thus, the shift of the Fermi surface upon deviation from the critical point receives two contributions: one analytic in $r$ and the other nonanalytic. Reexpressing the second contribution in terms of the correlation length, 


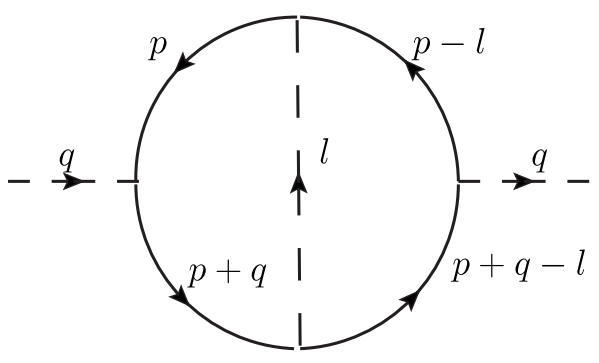

FIG. 4. Two-loop corrections to the boson self-energy.

$$
\Delta k=\frac{\alpha}{z-3} r e^{2}+\widetilde{C}_{k} \xi^{-2}+\delta
$$

where the coefficient $\widetilde{C}_{k}$ is expected to be universal. We would like to point out that the case $z=3$ has to be treated separately. In this situation one obtains

$$
\Delta k=\frac{\alpha r e^{2}}{2} \log \frac{r e^{2}}{\Lambda^{2}}=-\hat{C}_{k} \xi^{-2} \log (\Lambda \xi)+\delta
$$

with $\hat{C}_{k}$ again universal.

The value of the Fermi surface shift $\Delta k$ can be used to compute the compressibility, $\frac{\partial n}{\partial \mu}$, where $\mu$ is the physical chemical potential. Indeed, by Luttinger's theorem the change in density can be obtained as

$$
\delta n=\frac{N}{(2 \pi)^{2}} \int d s \Delta k(\theta),
$$

where the integral is over the circumference of the Fermi surface. The main question is how does the chemical potential enter our low-energy theory. If $\mu$ only couples to the operator $\psi^{\dagger} \psi$, renormalizing the value of $\delta$, then from Eqs. (4.59) and (4.61) we would conclude that the compressibility tends to a constant and has no interesting corrections near the quantum critical point. On the other hand, if the coupling $r$ has a nontrivial $\mu$ dependence, then we would conclude

$$
\begin{gathered}
\frac{\partial n}{\partial \mu}=\kappa_{0}+\kappa_{1} \xi^{z-3}, \quad z \neq 3, \\
\frac{\partial n}{\partial \mu}=\kappa_{0}+\hat{\kappa}_{1} \log \Lambda \xi, \quad z=3 .
\end{gathered}
$$

Note that for $z \geq 3$ the above forms imply that the compressibility diverges as we approach the critical point.

\section{ANOMALOUS EXPONENTS TO THREE LOOPS}

In this section, we evaluate the exponents $z$ and $\eta_{\psi}$ to three loop order. We find that the exponent $\eta_{\psi}$ is nonzero at this order. The value of $\eta_{\psi}$ is not suppressed in the large- $N$ limit. On the other hand, the dynamical critical exponent $z$ remains unrenormalized from its RPA value $z=3$ to this order. Moreover, in the large- $N$ limit, the boson self-energy acquires a finite correction of order $N^{3 / 2}$, which is larger than the bare value $(\operatorname{order} N)$. Finally, we find that the constant $\alpha$ in Eq. (4.30) associated with the shift of the Fermi surface
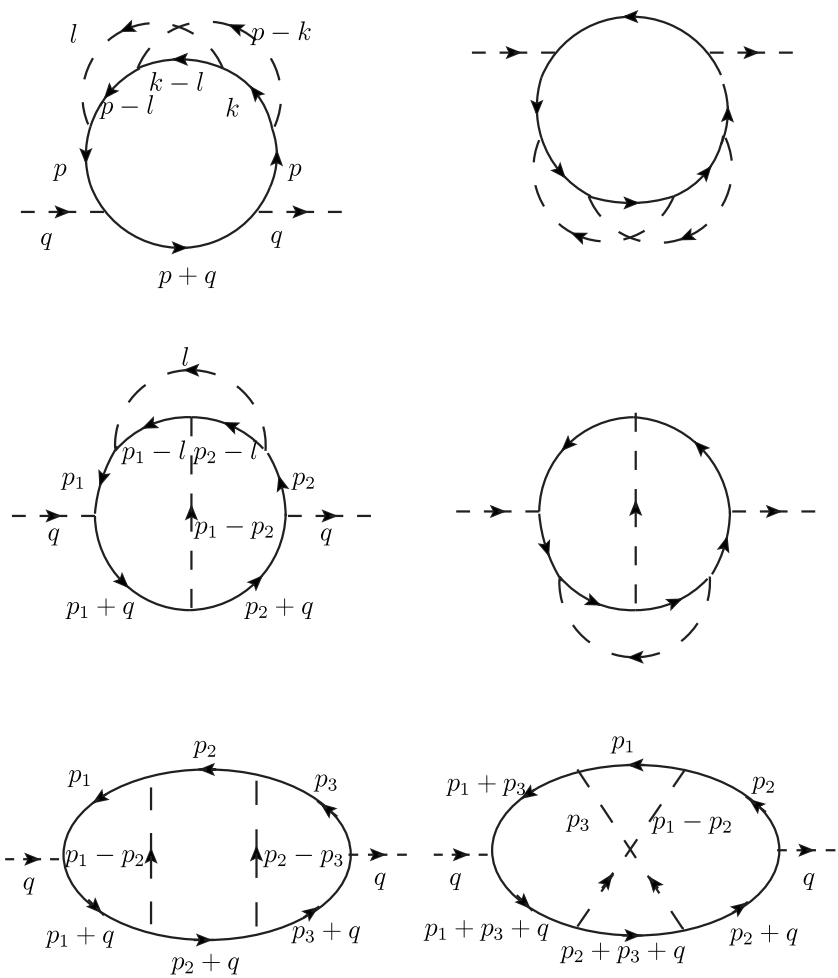

FIG. 5. Three-loop corrections to the boson self-energy with one fermion loop.

away from criticality is nonzero at three loop order. We note that the $N^{3 / 2}$ correction to the boson self-energy and the nonzero $\eta_{\psi}$ are only present for the Ising-nematic and spin-liquid universality classes, and are absent for the Ising ferromagnet transition.

\section{A. Dynamical critical exponent}

Let us first address the question of renormalization of $e^{2}$. At two loops the only correction to the static boson-selfenergy $\Pi\left(q_{\tau}=0, \vec{q}\right)$, which is not already taken into account by the solution to self-consistent Eliashberg equations is given in Fig. 4. However, this diagram vanishes when the external frequency is equal to zero. Indeed, as pointed out in Ref. 40, any diagram with fermions from a single patch, in which the fermion propagators involve a sum of two or less internal momenta, vanishes in the static limit (one picks the internal frequency with the largest absolute value and integrates over the corresponding $x$ component of the momentum. All poles will be in the same half plane). Actually, a calculation presented in Appendix B shows that the diagram in Fig. 4 vanishes for any external frequency and momentum.

The three loop corrections to $\Pi(q)$ are shown in Figs. 5 and 6. By the argument described above, all of these diagrams vanish when the external frequency is zero if all the fermions are from the same patch. Hence, the only nonzero corrections to $\Pi\left(q_{\tau}=0, \vec{q}\right)$ come from the Aslamazov-Larkintype diagrams, Fig. 6 , 


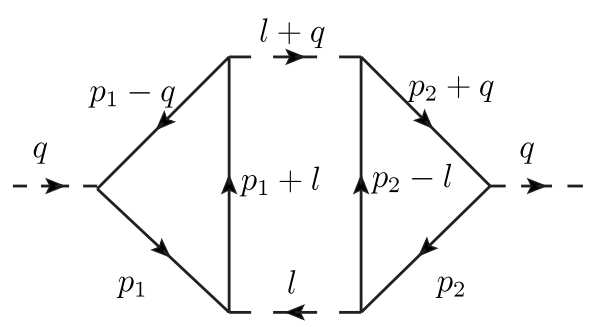

(a)

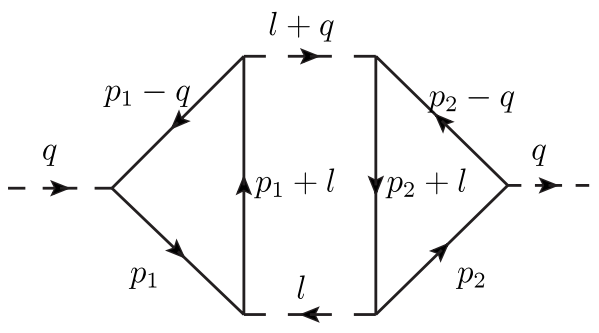

(b)

FIG. 6. Aslamazov-Larkin-type three-loop contributions to the boson self-energy.

$$
\begin{aligned}
\delta^{3} \Pi(q)= & -\frac{1}{2} \int \frac{d l_{\tau} d^{2} \vec{l}}{(2 \pi)^{3}} \Gamma^{3}[q, l,-(l+q)] \\
& \times \Gamma^{3}(-q,-l, l+q) D(l) D(l+q) .
\end{aligned}
$$

Here $\Gamma^{3}$ is the fermion-induced cubic boson vertex, which receives contribution from the two fermion patches,

$$
\begin{gathered}
\Gamma^{3}=\Gamma_{+}^{3}+\Gamma_{-}^{3}, \\
\Gamma_{s}^{3}\left(l_{1}, l_{2}, l_{3}\right)=N \lambda_{s}^{3}\left[f_{s}\left(l_{1}, l_{2}, l_{3}\right)+f_{s}\left(l_{2}, l_{1}, l_{3}\right)\right], \\
f_{s}\left(l_{1}, l_{2}, l_{3}\right)=\int \frac{d p_{\tau} d^{2} \vec{p}}{(2 \pi)^{3}} G_{s}(p) G_{s}\left(p-l_{1}\right) G_{s}\left(p+l_{2}\right) .
\end{gathered}
$$

The diagrams where the fermions in the two loops come from the same patch give a vanishing contribution to $\Pi\left(q_{\tau}\right.$ $=0, \vec{q})$. Thus, to three loops,

$$
\begin{aligned}
\delta^{3} \Pi\left(q_{\tau}=\right. & 0, \vec{q})=-\frac{1}{2} \int \frac{d l_{\tau} d^{2} \vec{l}}{(2 \pi)^{3}} \Gamma_{+}^{3}[q, l,-(l+q)] \\
& \times \Gamma_{-}^{3}(-q,-l, l+q) D(l) D(l+q)+(q \rightarrow-q) \\
= & -\lambda_{+}^{3} \lambda_{-}^{3} N^{2} \int \frac{d l_{\tau} d^{2} \vec{l}}{(2 \pi)^{3}}\left\{f _ { + } [ q , l , - ( l + q ) ] \left[f_{-}(-q,-l, l\right.\right. \\
& \left.\left.+q)+f_{-}(-q, l+q,-l)\right] D(l) D(l+q)\right\}+(q \rightarrow-q) .
\end{aligned}
$$

The two terms in brackets in the equation above originate, respectively, from diagrams in Figs. 6(a) and 6(b). Converting these diagrams into the double line representation of Ref. 40, we obtain Figs. 7(a) and 7(b). [We remark that the genus expansion of Ref. 40 was developed for a theory with only a

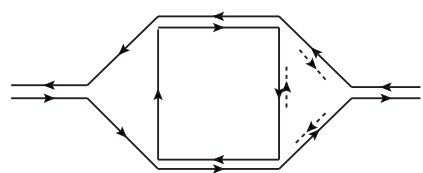

(a)

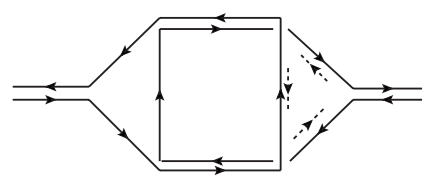

(b)

FIG. 7. Double line representation of Ref. 40 applied to the Aslamazov-Larkin diagrams in Fig. 6. The fermions in the two loops are assumed to come from opposite patches. We have reversed the directions of the fermion propagators from the second patch and the dotted arrows indicate the true directions of the fermion momenta.

single Fermi-surface patch. The extension to the present case of a pair of time reversed patches is simple: a reversal of the direction of loops with fermions from the second patch reduces the problem to that with one patch only. The diagrams in Fig. 7 have their lines reversed precisely in this way. The additional dotted arrow besides each propagator indicates the true direction of fermion momentum.] In this representation, the graph (a) contains a loop while the graph (b) does not. As a result, in the genus expansion of Ref. 40, the diagram in Fig. 6(a) is enhanced to $O(N)$ while the diagram in Fig. 6(b) is of $O(1)$. However, we will see that the diagrams are actually individually ultraviolet divergent, as a result the counting of Ref. 40 is inapplicable here. It turns out that the sum of the diagrams is UV finite and of $O\left(N^{3 / 2}\right)$.

We give details of the evaluation of Eq. (5.5) in Appendix $\mathrm{B}$, where we find

$$
\delta^{3} \Pi\left(q_{\tau}=0, \vec{q}\right)=C \lambda_{+} \lambda_{-} \frac{q_{y}^{2}}{e^{2}}
$$

In the large- $N$ limit, the coefficient $C$ is given by

$$
C \approx-0.09601 N^{3 / 2}, \quad N \rightarrow \infty
$$

while for the physical value $N=2$,

$$
C \approx-0.04455, \quad N=2
$$

The $N^{3 / 2}$ behavior in Eq. (5.7) indicates a breakdown of the genus expansion of Ref. 40. Moreover, since this correction is parametrically larger than the tree level value, the existence of the large- $N$ limit of the theory is cast into doubt. In particular, it is not clear if there are higher loop graphs with even stronger divergences in the large- $N$ limit. Moreover, we expect contributions to the bosonic self-energy analytic in $q_{y}$ to be generated from kinematic regimes involving the whole Fermi surface and not just the two Fermi patches. Such analytic contributions might also exhibit anomalous scaling with $N$. 


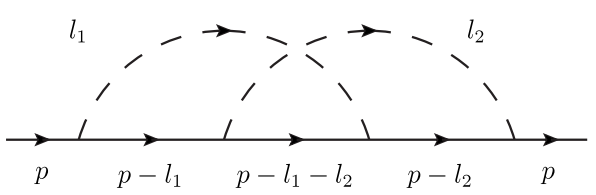

FIG. 8. Fermion self-energy at two loops.

Note that there is no logarithmic dependence on $\Lambda / \mu$ in Eq. (5.6) and so we have $z=3$ at this order. For the physical value of $N=2$, the finite three-loop correction turns out to be rather small numerically.

\section{B. Fermion anomalous dimension}

The Feynman diagrams for the fermion self-energy up to three loop order are shown in Figs. 8-10. By reasons explained in the previous section, the diagrams in Figs. 8 and 9 vanish when the external frequency is zero and, hence, do not contribute to the fermion anomalous dimension.

Thus, the only fermion self-energy diagrams that can give UV divergences are shown in Fig. 10. Actually, the diagram in Fig. 10(a) is zero since the polarization correction in Fig. 4 vanishes. Thus, we only need to consider the two diagrams in Figs. 10(b) and 10(c). For these graphs to be UV divergent, the fermions running in the loop and the external fermions must come from different patches. The diagram in Fig. 10(b) contains two loops in the double line representation [Fig. 11(a)] and is expected to be of order $1 / N$ while the one in Fig. 10(c) has no loops in the double line representation [Fig. 11(b)] and, hence, is expected to scale as $1 / N^{2}$.

A calculation presented in Appendix B gives the UV divergent contribution,

$$
\delta^{3 b} \Sigma_{+}(\omega=0, \vec{p})=\lambda_{+} \lambda_{-} J_{b}\left(p_{x}+p_{y}^{2}\right) \log \left(\frac{\Lambda_{y}}{\left|p_{x}+p_{y}^{2}\right|^{1 / 2}}\right),
$$
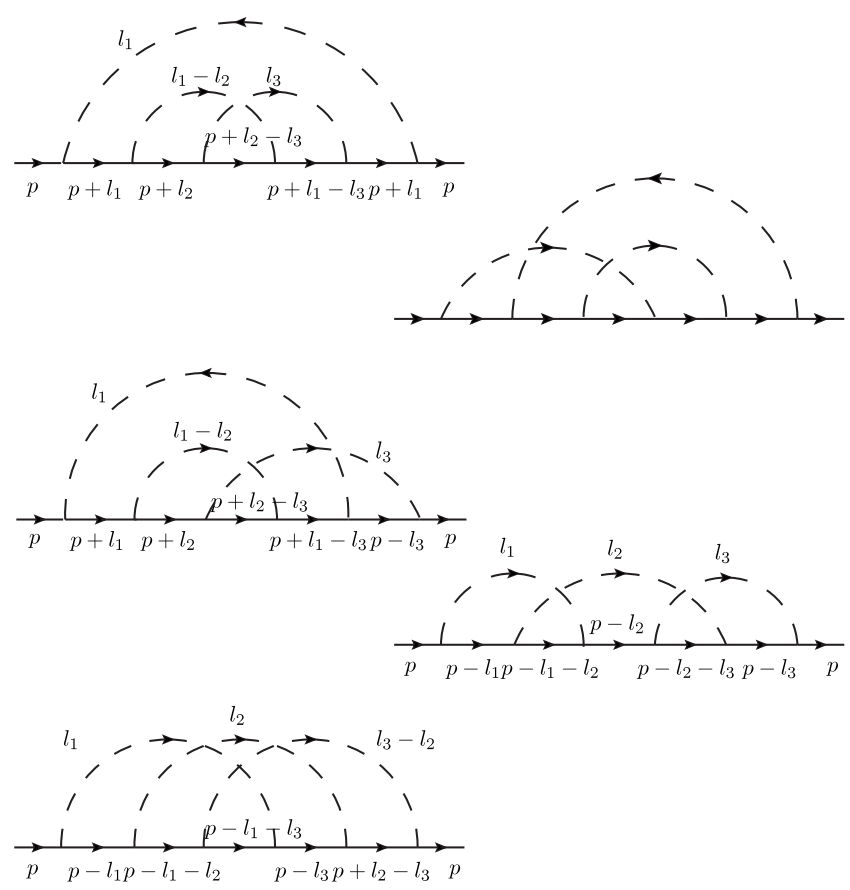

FIG. 9. Three-loop fermion self-energy diagrams with no fermion loops.

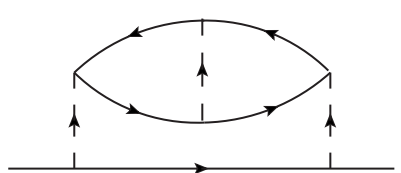

(a)

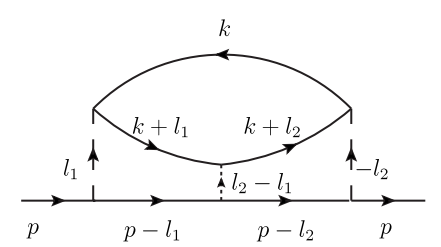

(b)

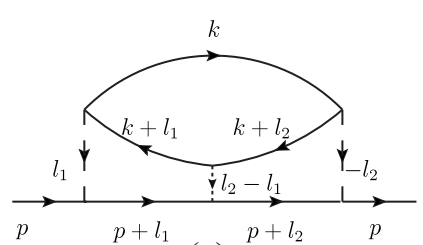

(c)
FIG. 10. Three-loop fermion self-energy diagrams with one fermion loop.

$$
\begin{aligned}
\delta^{3 c} \Sigma_{+}(\omega= & 0, \vec{p}) \\
= & \delta^{3 c} \Sigma_{+}(\omega=0, \vec{p}=0)+\lambda_{+} \lambda_{-} J_{c} \\
& \times\left(p_{x}+p_{y}^{2}\right) \log \left(\frac{\Lambda_{y}}{\left|p_{x}+p_{y}^{2}\right|^{1 / 2}}\right) .
\end{aligned}
$$

The constant $J_{b}$ is independent of $N$ and given numerically by

$$
J_{b} \approx 0.1062 \text {. }
$$

On the other hand, the constant $J_{c}$ is $N$ dependent. For $N$ $=2$ we obtain,

$$
J_{c} \approx-0.03795, \quad N=2
$$

while in the large- $N$ limit,

$$
J_{c} \approx \frac{9}{4 \pi^{2} N^{2}} \log ^{3} N, \quad N \rightarrow \infty .
$$

Notice that there is no $1 / N$ suppression in Eq. (5.9). A way to interpret this, is that the diagram is really of order $1 / N$ (as the genus expansion predicts), however, it is a function of $N\left(p_{x}+p_{y}^{2}\right)$. Indeed, recall that the genus expansion assumes $N\left(p_{x}+p_{y}^{2}\right) \sim 1$. However, the UV divergent piece of the diagram cannot depend on the magnitude of $p_{x}+p_{y}^{2}$ and is valid

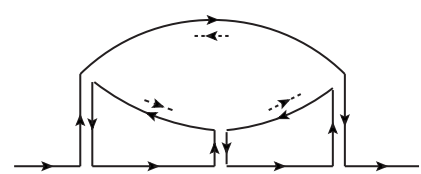

(a)

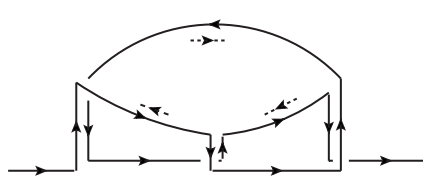

(b)

FIG. 11. Double line representation of fermion self-energy diagrams in Figs. 10(b) and 10(c), as in Fig. 7. The external fermions and the fermions inside the loop are assumed to come from opposite patches. 
for any external momentum or frequency. On the other hand, the infrared scale under the log is expected to become $\omega^{1 / 3}$ once $\omega \gg N^{3 / 2}\left|p_{x}+p_{y}^{2}\right|^{3 / 2}$. Also observe that up to a logarithmic enhancement, the nonplanar diagram in Fig. 10(c) [Fig. $11(\mathrm{~b})]$ is of order $1 / N^{2}$, as expected from the genus expansion.

Note that the UV divergence in Eqs. (5.9) and (5.10) is logarithmic, as expected from power counting, and comes from a region where both internal momenta and frequencies diverge in accordance with the scaling, Eq. (4.1). This is unlike the anomalous linear divergences of the AslamazovLarkin diagrams that occur when the internal momenta $q_{y}$ are of order of external momenta while internal $q_{x}, q_{\tau}$ diverge.

Thus, to three-loop order,

$$
\begin{gathered}
\delta^{3} \Sigma_{+}(\omega=0, \vec{p})=\delta^{3} \Sigma_{+}(\omega=0, \vec{p}=0) \\
+\lambda_{+} \lambda_{-} J\left(p_{x}+p_{y}^{2}\right) \log \left(\frac{\Lambda_{y}}{\left|p_{x}+p_{y}^{2}\right|^{1 / 2}}\right), \\
J=J_{b}+J_{c} \approx \begin{cases}0.06824 & N=2 \\
0.10619 & N=\infty .\end{cases}
\end{gathered}
$$

Although the self-energy correction, Eq. (5.14), is not parametrically suppressed compared to the bare value even when $N=\infty$, it appears to be suppressed numerically. Thus, we may estimate,

$$
\begin{aligned}
& Z_{\psi}=1-\lambda_{+} \lambda_{-} J \log \Lambda / \mu, \\
& \eta_{\psi}=\lambda_{+} \lambda_{-} J= \pm 0.06824,
\end{aligned}
$$

where the upper sign refers to the Ising-nematic transition and the lower sign to the spin liquid and we have used the value of $J$ at $N=2$.

\section{Fermi-surface shift}

We now evaluate the coefficient $\alpha$, Eq. (4.30), associated with the renormalization of chemical potential $\delta$ away from criticality. This coefficient can be obtained from the insertion of the $\phi^{2}$ operator into the two-point fermion Green's function at criticality. By setting all external frequencies to zero, we find that at three-loop order the only UV divergent contribution can originate from the diagrams in Figs. 10(b) and $10(\mathrm{c})$ with the $\phi^{2}$ operator inserted into the boson propagators. The details of the calculation are presented in Appendix B. We find

$$
\delta^{3} \frac{\partial \Sigma}{\partial r}=J_{r} e^{2} \log \Lambda_{y}
$$

with

$$
\begin{gathered}
J_{r}=0.00208, \quad N=2, \\
J_{r} \sim O\left(\frac{1}{N^{3}}\right), \quad N \rightarrow \infty .
\end{gathered}
$$

Absorbing this divergence into the chemical potential,

$$
Z_{r \delta}=J_{r} \log \Lambda_{y} / \mu
$$

and

$$
\alpha=J_{r} .
$$

Thus, the $\phi^{2}$ operator mixes with the $\psi^{\dagger} \psi$ operator. If the dynamical critical exponent $z=3$, this leads to a logarithmic divergence of the compressibility, Eq. (4.64). Note that the magnitude of the mixing $\alpha$ is suppressed in the large- $N$ limit and is also numerically small for $N=2$.

\section{CONCLUSIONS}

This paper has presented the scaling properties of the field theory in Eq. (2.7) which describes a number of problems involving the breakdown of Landau Fermi-liquid theory at all points on a two-dimensional Fermi surface. The main motivation was provided by the quantum phase transition caused by the onset of Ising-nematic order, which reduces the point-group symmetry from square to rectangular. However our theory also directly applies or can be generalized to breaking of other point-group and/or time-reversal symmetries, and these were described in Sec. II. One of these cases is the "circulating current" order parameter of Simon and Varma. ${ }^{33,47,48}$ Apart from applications to quantum critical points, our theory also described non-Fermi-liquid phases associated with spin liquids ${ }^{57,58}$ or algebraic charge liquids, ${ }^{59-61}$ which have Fermi surfaces coupled to U(1) gauge fields.

Our critical theory was formulated in terms of a timereversed pair of patches on the Fermi surface, centered at the wave vectors $\pm \vec{k}_{0}$ (see Fig. 1 ). The value of $\vec{k}_{0}$ was determined by requiring that the tangent to the Fermi surface at $\vec{k}_{0}$ be parallel to the wave vector $\vec{q}$ carried by the order parameter insertion in the correlation function being computed. However, in general, there is nothing special about the point $\vec{k}_{0}$, and neighboring points on the Fermi surface should behave in a similar manner. This key feature was implemented in our theory by the rotational symmetry discussed in Sec. IV A, and the identities (4.5) and (4.6), which show that the Green's function remains invariant as we move along the Fermi surface.

We emphasize that although we have critical theories associated with every pair of points on the Fermi surface, the Lagrangian (2.7) and all the fields are $2+1$ dimensional, i.e., $\phi$ and $\psi_{\sigma}$ are integrated over arbitrary functions of $x, y$, and $\tau$. Thus, as we noted earlier, our approach and results differ from studies using a "tomographic" representations of the Fermi surface, in which every point on the Fermi surface is described by a 1+1-dimensional field theory. ${ }^{27,28,41-45}$ Our $2+1$-dimensional representation leads to a redundancy in our description of the degrees of freedom and the identities of Sec. IV A ensure the consistency of this redundant description.

Our main results include the scaling relations for the order parameter susceptibility in Eq. (4.45) and for the fermion Green's function in Eq. (4.50). These are associated with only two independent exponents, the dynamic scaling exponent $z$, and the fermion anomalous dimension $\eta_{\psi}$. The correlation length exponent $\nu$ was given by exact scaling relation in Eq. (4.40) while the susceptibility exponent $\gamma=1$. For the spin-liquid case, Fermi-liquid arguments were 
made $49,50,52-54,56$ suggesting that $z=3$; we found $z=3$ to threeloop order in Sec. V, although we did not prove this to all orders, and our scaling theory is compatible with a general value of $z$. Our three-loop computation also gave a nonzero value of $\eta_{\psi}$ with opposite signs for the Ising-nematic and spin-liquid cases. In the case of the nematic transition, a nonzero positive $\eta_{\psi}$ implies the suppression of the electron tunneling density of states, Eq. (4.58). Another striking effect that we find for the case of a nematic transition is the powerlaw divergence of the compressibility for $z>3$, which turns into a logarithmic divergence if $z=3$.

Our scaling results were expressed in terms of correlators of the fermionic field $\psi_{+\sigma}$ carrying momentum $\vec{q}$ as measured from the point $\vec{k}_{0}$ from the Fermi surface, implying from Eq. (2.3) that the electron $c_{\sigma}$ has momentum $\vec{k}_{0}+\vec{q}$ (and similarly for $\left.\psi_{-\sigma}\right)$. However, note that (after appropriate rescaling of momenta, and for a circular Fermi surface) $|\vec{k}|-k_{F} \approx q_{x}+q_{y}^{2}$. Thus the identity (4.6) implies that the scaling function, Eq. (4.50), for the two-point fermion Green's function depends only on $|\vec{k}|-k_{F}$. This is similar to the dependence found in other treatments, e.g., in the recent critical theories ${ }^{62-65}$ obtained by applying the AdS/CFT duality to fermions propagating near a Reissner-Nordstrom black hole. The latter theories, in their current classical gravity formulation, find ${ }^{64} \eta_{\psi}$ $=0$.

It is also interesting to compare the structure of the critical theory in the AdS/CFT framework to that found here. We have an infinite set of 2+1-dimensional field theories labeled by pairs of momenta on a one-dimensional Fermi surface, i.e., a $\mathrm{S}^{1} / \mathbb{Z}_{2}$ set of $2+1$-dimensional field theories. In the low-energy limit, the AdS/CFT approach yields ${ }^{64}$ a $\mathrm{AdS}_{2}$ $\times \mathbb{R}^{2}$ geometry: this can be interpreted as an infinite set of chiral 1+1-dimensional theories labeled by a $\mathbb{R}^{2}$ set of twodimensional momenta $\vec{k}$. It is notable, and perhaps significant, that both approaches have an emergent dimension not found in the underlying degrees of freedom. We began with a $2+1$-dimensional Hamiltonian and ended up with a $\mathrm{S}^{1} / \mathbb{Z}_{2}$ set of 2+1-dimensional field theories. In AdS/CFT, there is the emergent radial direction representing energy scale. These emergent dimensions imply redundant descriptions and require associated consistency conditions: we explored such consistency conditions in Sec. IV A while in AdS/CFT the consistency conditions are Einstein's equations representing the renormalization-group flow under changes in energy scale. It would be interesting to see if fluctuations about the classical gravity theory yield corrections to the $\mathrm{AdS}_{2} \times \mathrm{R}^{2}$ geometry which clarify the connection to our theory.

In the analysis of the spin-liquid problem, Ref. 40 considered a single patch of the Fermi surface, and argued that the $1 / N$ expansion should be organized by the genus of the Feynman graph (after the propagators are written in a suitable double line representation and the graph is interpreted as lying on a two-dimensional surface). In our two-patch theory here, we have shown that this genus counting is violated. This is the implication of the $N^{3 / 2}$ dependence of the boson self-energy in Eq. (5.6). In fact, at present, it is not clear how to take the large- $N$ limit of the theory. On the other hand, for the physical value $N=2$, we found that the higher-loop contributions are numerically small, which suggests that the critical exponents are close to the Hertz mean-field values. However, because the loopwise expansion does not possess even a formal expansion parameter, it is not clear if there is a systematic way to extract corrections to the mean-field exponents. Thus, our value of the fermion anomalous dimension $\eta_{\psi}$, Eq. (5.16), should be regarded as an estimate only.

\section{ACKNOWLEDGMENTS}

We thank E. Altman, G. Baskaran, A. Chubukov, E. Fradkin, B. I. Halperin, S. Hartnoll, Y.-B. Kim, S. A. Kivelson, S.-S. Lee, W. Metzner, Y. Oreg, D. Scalapino, A. Schiller, T. Senthil, R. Shankar (IMSc), R. Shankar (Yale), A. Stern, L. Taillefer, and C. Xu for useful discussions. This research was supported by the National Science Foundation under Grant No. DMR-0757145, by the FQXi foundation, and by a MURI grant from AFOSR.

\section{APPENDIX A: DECOUPLING OF NONCOLLINEAR MOMENTA}

In this section we will argue that the fluctuations of the order parameter at noncollinear momenta effectively decouple. We focus for simplicity on the case of an Isingnematic transition. We follow the standard Hertz approach, integrating out the fermions to obtain an effective action for $\phi$,

$$
\begin{aligned}
S[\phi]= & \sum_{n=2}^{\infty} \frac{1}{n !} \int d^{D} x_{1} \cdots d^{D} x_{n} \Gamma^{n}\left(x_{1}, x_{2}, \ldots, x_{n}\right) \\
& \times \phi\left(x_{1}\right) \phi\left(x_{2}\right) \cdots \phi\left(x_{n}\right) .
\end{aligned}
$$

The $n$-point effective vertex $\Gamma^{n}$ is given by

$$
\begin{aligned}
\Gamma^{n}\left(q_{1}, q_{2}, \ldots, q_{n}\right)= & \frac{N}{n} f^{n}\left(q_{1}, q_{2}, \ldots, q_{n}\right) \\
& + \text { permutations of } q_{1}, q_{2}, \ldots, q_{n}
\end{aligned}
$$

with

$$
f^{n}\left(q_{1}, q_{2}, \ldots, q_{n}\right)=\int \frac{d k_{\tau} d^{2} k}{(2 \pi)^{3}} \prod_{i=0}^{n-1}\left[G\left(k+l_{i}\right) d_{\vec{k}+\left(\vec{l}_{i}+\vec{l}_{i+1}\right) / 2}\right],
$$

where $l_{i}=\sum_{j=1}^{i} q_{j}$. For now we work with "undressed" propagators,

$$
G(\omega, \vec{k})=\frac{1}{-i \omega+v_{F}(\theta) k}
$$

with $k$-the distance to the Fermi surface and $v_{F}(\theta)$-the local Fermi velocity. As is well known, for $\omega \ll v_{F}|\vec{q}|$ and $|\vec{q}| \ll k_{F}$ the two-point vertex has a Landau-damped form,

$$
\Gamma^{2}(\omega, \vec{q})=N\left[\gamma(\hat{q}) \frac{|\omega|}{|\vec{q}|}+\frac{\vec{q}^{2}}{e^{2}}+r\right]
$$

where the coefficient of the nonanalytic term $\gamma(\hat{q})$ $=K d^{2} /\left(2 \pi v_{F}^{2}\right)$ with the Fermi-surface curvature radius $K$, 
Fermi-velocity $v_{F}$, and form-factor $d$ evaluated at the point on the Fermi surface to which $\vec{q}$ is tangent. On the other hand, the coefficients of the analytic terms $r$ and $1 / e^{2}$ come from the entire Fermi surface.

If we truncate the series, Eq. (A1), at the quadratic order,

$$
S_{2}=\frac{N}{2} \int \frac{d \omega d^{2} \vec{q}}{(2 \pi)^{3}}\left[\gamma(\hat{q}) \frac{|\omega|}{|\vec{q}|}+\frac{\vec{q}^{2}}{e^{2}}+r\right]|\phi(\vec{q}, \omega)|^{2}
$$

then at the critical point $r=0$ the action, Eq. (A6), is invariant under the scale transformation,

$$
\phi(\vec{x}, \tau) \rightarrow s^{3 / 2} \phi\left(s \vec{x}, s^{3} \tau\right)
$$

Note that here, in contrast to Eq. (4.1), all components of $\vec{q}$ are scaled in the same way as we are not studying the effects of fluctuations with collinear wave vectors. We can regard the terms in Eq. (A1) with $n>2$ as perturbations to the Hertz action, Eq. (A6). Hertz noted that if the effective vertices $\Gamma^{n}$ possess a regular expansion in frequencies and momenta, such that the corresponding operators can be represented as polynomials in the order parameter $\phi$ and its derivatives, then the perturbations with $n>2$ are irrelevant due to the large effective dimensionality, $D_{e f f}=d+z=5$ with $d=2$ - spatial dimension and $z=3$ - the dynamical critical exponent. Indeed, the perturbation $\int d^{2} \vec{x} d \tau \phi^{n}(x)$ scales as $s^{3 n / 2-5}$ under Eq. (A7) (in the special case $n=3$, the operator $\phi^{3}$ is actually prohibited by the $90^{\circ}$ lattice rotation symmetry. The lowest dimension local operators with three powers of $\phi$ that are allowed by symmetry are $\phi\left[\left(\partial_{x} \phi\right)^{2}-\left(\partial_{y} \phi\right)^{2}\right]$ in the $d_{x^{2}-y^{2}}$ case and $\phi \partial_{x} \phi \partial_{y} \phi$ in the $d_{x y}$ case, which scale as $s^{3 / 2}$ ).

However, due to the presence of low-energy excitations on the Fermi surface there is no reason to expect that the effective vertices $\Gamma^{n}$ would possess a regular expansion in momenta. Indeed, we have already seen that the two-point vertex has the nonanalytic Landau-damped form, Eq. (A5). As we now show, similar nonanalyticities occur in the higher-order vertices.

Let us estimate the vertices, Eq. (A2), when the external frequencies and momenta obey the Hertz scaling, Eq. (A7), $\omega \sim|\vec{q}|^{3}, \vec{q} \rightarrow 0$. In this regime,

$$
\begin{aligned}
f^{n}\left(q_{1}, q_{2}, \ldots, q_{n}\right)= & \int \frac{d k_{\tau} d k d \theta}{(2 \pi)^{3}}\left|\frac{d \vec{k}_{F}}{d \theta}\right| d(\theta)^{n} \\
& \times \prod_{i=0}^{n-1} \frac{1}{-i\left(k_{\tau}+l_{i \tau}\right)+v_{F}(\theta)\left[k+\hat{v}_{F}(\theta) \cdot \vec{l}_{i}\right]}
\end{aligned}
$$

Let us perform the integral over $k$. Observe that if $\left|k_{\tau}\right|>\Omega$ with $\Omega=\max _{i}\left|l_{i \tau}\right|$ then the integral vanishes as all the poles of the integrand are in the same half plane. Thus, the range of the internal frequency is limited by the external ones. With this in mind,

$$
\begin{aligned}
f^{n}\left(q_{1}, q_{2}, \ldots, q_{n}\right)= & i \int_{\left|k_{\tau}\right|<\Omega} \frac{d k_{\tau}}{2 \pi} \int \frac{d \theta}{2 \pi}\left|\frac{d \vec{k}_{F}}{d \theta}\right| \frac{d(\theta)^{n}}{v_{F}(\theta)} \\
& \times \sum_{j=0}^{n-1} \vartheta\left(k_{\tau}+l_{j \tau}\right) \\
& \times \prod_{i=0, i \neq j}^{n-1} \frac{1}{-i\left(l_{i \tau}-l_{j \tau}\right)+\hat{v}_{F}(\theta) \cdot\left(\vec{l}_{i}-\vec{l}_{j}\right)}
\end{aligned}
$$

where we have used the symbol $\vartheta$ for the step function to avoid confusion with the angular variable $\theta$. Now, since $q_{\tau}$ $\sim|\vec{q}|^{3} /\left(\gamma e^{2}\right) \ll v_{F}|\vec{q}|$, for general $\theta$ we can ignore the frequency dependence in the denominator of Eq. (A9). Then the angular integration yields a factor of $\mathcal{O}(1)$ and the integral over $k_{\tau}$ yields a factor of external frequency so that

$$
\Gamma^{n}\left(q_{1}, q_{2}, \ldots, q_{n}\right) \sim \frac{q_{\tau}}{|\vec{q}|^{n-1}} .
$$

Note that the momentum dependence in Eq. (A10) is far from analytic. Also, note that for $n=2$ the result is consistent with the standard Landau damping.

The only possible caveat to the estimate, Eq. (A10), is associated with regions of angular integration where $\hat{v}_{F}(\theta) \cdot\left(\vec{l}_{i}-\vec{l}_{j}\right) \rightarrow 0$, i.e., some combination of external momenta becomes tangent to the Fermi surface. Then the angular integration acquires poles just off the real axis with the imaginary parts of the poles provided by the frequency dependence in the denominator of Eq. (A9). As long as the real parts of the poles do not coalesce, i.e., no two momenta $\vec{l}_{i}$ $-\vec{l}_{j}$ and $\vec{l}_{i^{\prime}}-\vec{l}_{j}$ are collinear, the angular integration still yields a factor of $\mathcal{O}(1)$ and the estimate, Eq. (A10), remains correct. This is the regime that we are considering in the present appendix. The rest of the paper is devoted to the opposite limit, where all the external momenta are nearly collinear and the angular integral in Eq. (A9) is dominated by the vicinity of two antipodal points on the Fermi surface to which the external momenta are tangent. This observation motivates the introduction of the two patch theory in Sec. II and all the subsequent development of the present work.

Returning to the noncollinear regime, upon combining Eq. (A10) with the Hertz scaling, Eq. (A7), we conclude that the $n$th term in the series, Eq. (A1), scales as $s^{n / 2-1}$. Therefore, all terms with $n>2$ represent nonlocal irrelevant perturbations, which confirms that the fluctuations with noncollinear momenta decouple.

We would like to point out that the argument above still holds if one dresses the fermion propagator by the one-loop self-energy, $\Sigma(\omega, k) \sim-i \operatorname{sgn} \omega|\omega|^{2 / 3}$. This modifies the frequency dependence in the denominator of Eq. (A9) via, $-i\left(l_{i \tau}-l_{j \tau}\right) \rightarrow \Sigma\left(k_{\tau}+l_{i \tau}\right)-\Sigma\left(k_{\tau}+l_{j \tau}\right)$. However, since $\Sigma(\omega)$ $\ll v_{F}|\vec{q}|$ for typical $\omega \sim|\vec{q}|^{3}$, the estimate, Eq. (A10), is still correct. 


\section{APPENDIX B: COMPUTATION OF FEYNMAN DIAGRAMS}

Here we provide some details of the computations of the diagrams in Sec. V.

\section{Boson self-energy}

We begin by evaluating the two-loop polarization correction in Fig. 4,

$$
\delta^{2} \Pi(q)=N \sum_{s} \int \frac{d p_{\tau} d^{2} p}{(2 \pi)^{3}} \frac{d l_{\tau} d^{2} l}{(2 \pi)^{3}} D(l) G_{s}(p) G_{s}(p+q) G_{s}(p-l) G_{s}(p+q-l) .
$$

The contributions to the integral from the two patches are equal. Thus, integrating over $p_{x}, l_{x}$ we obtain,

$$
\delta^{2} \Pi(q)=2 N \int \frac{d p_{\tau} d p_{y}}{(2 \pi)^{2}} \frac{d l_{\tau} d l_{y}}{(2 \pi)^{2}} D(l) \frac{\theta\left(p_{\tau}\right)-\theta\left(p_{\tau}+q_{\tau}\right)}{\frac{i c_{f}}{N}(\{p\}-\{p+q\})+2 q_{y} p_{y}+q_{x}+q_{y}^{2}} \times \frac{\theta\left(l_{\tau}-p_{\tau}\right)-\theta\left(l_{\tau}-p_{\tau}-q_{\tau}\right)}{\frac{i c_{f}}{N}(\{l-p-q\}-\{l-p\})+2 q_{y}\left(p_{y}-l_{y}\right)+q_{x}+q_{y}^{2}},
$$

where here and below we use the notation $\{p\}=\operatorname{sgn}\left(p_{\tau}\right)\left|p_{\tau}\right|^{2 / 3}$. We observe that the poles of the $p_{y}$ integral are always in the same half plane. Thus, $\delta^{2} \Pi(q)=0$. This is consistent with Ref. 50, which found that the two-loop corrections to Eq. (3.3) are suppressed by factors of $|\omega|^{2 / 3}$ or $|\omega| /\left|q_{y}\right| \sim|\omega|^{2 / 3}$.

Now, let us proceed to compute the Aslamazov-Larkin diagrams, Fig. 6. We begin by evaluating the three point function $f_{s}[q, l,-(l+q)]$ in Eq. (5.4). Note that $f_{-}[q, l,-(l+q)]=f_{+}\left[P_{x} q, P_{x} l,-P_{x}(l+q)\right]$, where $P_{x}\left(k_{0}, k_{x}, k_{y}\right)=\left(k_{0},-k_{x}, k_{y}\right)$. The calculation of $f$ is simplified when $q_{\tau}=0$. Then, performing the integral over $p_{x}$ and, subsequently, $p_{y}$, in Eq. (5.4),

$$
\begin{aligned}
f_{+}[q, l,-(l+q)] & =\int \frac{d p_{\tau} d p_{y}}{(2 \pi)^{2}} \frac{i\left[\theta\left(p_{\tau}+l_{\tau}\right)-\theta\left(p_{\tau}\right)\right]}{\frac{i c_{f}}{N}[\{p+l\}-\{p\}]-l_{x}-2 l_{y} p_{y}-l_{y}^{2} \frac{i c_{f}}{N}[\{p+l\}-\{p\}]-q_{x}-l_{x}-2\left(q_{y}+l_{y}\right) p_{y}+q_{y}^{2}-l_{y}^{2}} \\
& =\frac{1}{2 q_{y}} \int \frac{d p_{\tau}}{2 \pi} \frac{\left|\theta\left(p_{\tau}+l_{\tau}\right)-\theta\left(p_{\tau}\right)\right|\left[\theta\left(l_{y}\right)-\theta\left(q_{y}+l_{y}\right)\right]}{\frac{-i c_{f}}{N}[\{p+l\}-\{p\}]+l_{x}-\frac{q_{x}}{q_{y}} l_{y}+l_{y}\left(q_{y}+l_{y}\right)} .
\end{aligned}
$$

Thus,

$$
\begin{aligned}
& \delta^{3} \Pi\left(q_{\tau}=0, \vec{q}\right)=\frac{\lambda_{+} \lambda_{-} N^{2}}{4 q_{y}^{2}} \int \frac{d l_{\tau} d^{2} \vec{l}}{(2 \pi)^{3}} \frac{d p_{\tau}}{2 \pi} \frac{d p_{\tau}^{\prime}}{2 \pi} D(l) D(l+q) \frac{\left|\theta\left(p_{\tau}+l_{\tau}\right)-\theta\left(p_{\tau}\right)\right|\left|\theta\left(p_{\tau}^{\prime}+l_{\tau}\right)-\theta\left(p_{\tau}^{\prime}\right)\right|\left|\theta\left(l_{y}\right)-\theta\left(l_{y}+q_{y}\right)\right|}{\frac{-i c_{f}}{N}(\{p+l\}-\{p\})+l_{x}-\frac{q_{x}}{q_{y}} l_{y}+l_{y}\left(q_{y}+l_{y}\right)} \\
& \times\left(\frac{1}{\frac{-i c_{f}}{N}\left(\left\{p^{\prime}+l\right\}-\left\{p^{\prime}\right\}\right)-l_{x}+\frac{q_{x}}{q_{y}} l_{y}-l_{y}\left(q_{y}+l_{y}\right)}-\frac{1}{\frac{-i c_{f}}{N}\left(\left\{p^{\prime}+l\right\}-\left\{p^{\prime}\right\}\right)-l_{x}+\frac{q_{x}}{q_{y}} l_{y}+l_{y}\left(q_{y}+l_{y}\right)}\right)+(q \rightarrow-q) .
\end{aligned}
$$

Finally, integrating over $l_{x}$,

$$
\begin{aligned}
\delta^{3} \Pi\left(q_{\tau}=\right. & 0, \vec{q})=\frac{\lambda_{+} \lambda_{-} N^{2}}{4 q_{y}^{2}} \int \frac{d l_{\tau} d l_{y}}{(2 \pi)^{2}} \frac{d p_{\tau}}{2 \pi} \frac{d p_{\tau}^{\prime}}{2 \pi} D(l) D(l+q)(i) \operatorname{sgn}\left(l_{\tau}\right)\left|\theta\left(p_{\tau}+l_{\tau}\right)-\theta\left(p_{\tau}\right)\right|\left|\theta\left(p_{\tau}^{\prime}+l_{\tau}\right)-\theta\left(p_{\tau}^{\prime}\right)\right|\left|\theta\left(l_{y}\right)-\theta\left(l_{y}+q_{y}\right)\right| \\
& \times\left(\frac{1}{\frac{-i c_{f}}{N}\left(\{p+l\}-\{p\}+\left\{p^{\prime}+l\right\}-\left\{p^{\prime}\right\}\right)}-\frac{1}{\frac{-i c_{f}}{N}\left(\{p+l\}-\{p\}+\left\{p^{\prime}+l\right\}-\left\{p^{\prime}\right\}\right)+2 l_{y}\left(q_{y}+l_{y}\right)}\right)+(q \rightarrow-q) .
\end{aligned}
$$

The integral is invariant under $q \rightarrow-q$. Moreover, the integrals in the regions $l_{\tau}>0$ and $l_{\tau}<0$ are related by complex conjugation. Thus, 


$$
\begin{aligned}
\delta^{3} \Pi\left(q_{\tau}=\right. & 0, \vec{q})=-\frac{\lambda_{+} \lambda_{-} N}{q_{y}^{2}} \int_{0}^{\infty} \frac{d l_{\tau}}{2 \pi} \int_{0}^{l_{\tau}} \frac{d p_{\tau}}{2 \pi} \int_{0}^{l_{\tau}} \frac{d p_{\tau}^{\prime}}{2 \pi} \int_{0}^{\left|q_{y}\right|} \frac{d l_{y}}{2 \pi} \frac{1}{c_{b} \frac{l_{\tau}}{l_{y}}+\frac{l_{y}^{2}}{e^{2}} \frac{1}{c_{b} \frac{l_{\tau}}{\left|q_{y}\right|-l_{y}}+\frac{\left(\left|q_{y}\right|-l_{y}\right)^{2}}{e^{2}}}} \\
& \times\left\{\frac{1}{c_{f}\left[(l-p)_{\tau}^{2 / 3}+p_{\tau}^{2 / 3}+\left(l-p^{\prime}\right)_{\tau}^{2 / 3}+p_{\tau}^{\prime 2 / 3}\right]}-\frac{c_{f}\left[(l-p)_{\tau}^{2 / 3}+p_{\tau}^{2 / 3}+\left(l-p^{\prime}\right)_{\tau}^{2 / 3}+p_{\tau}^{\prime 2 / 3}\right]}{c_{f}^{2}\left[(l-p)_{\tau}^{2 / 3}+p_{\tau}^{2 / 3}+\left(l-p^{\prime}\right)_{\tau}^{2 / 3}+p_{\tau}^{\prime 2 / 3}\right]^{2}+4 N^{2} l_{y}^{2}\left(\left|q_{y}\right|-l_{y}\right)^{2}}\right\} .
\end{aligned}
$$

Notice that the integral over $l_{y}$ is bounded by the external momentum $q_{y}$. This leads to a violation of the naive power counting, which would predict that each diagram in Fig. 6 has a superficial degree for divergence $\Lambda_{y}^{2} \sim \Lambda_{\tau}^{2 / 3}$. Instead, we find that for $l_{\tau} \rightarrow \infty$, the two diagrams behave as

$$
\delta^{3 a} \Pi(0, \vec{q})=-\delta^{3 b} \Pi(0, \vec{q}) \sim-\lambda_{+} \lambda_{-} N\left|q_{y}\right|\left(\frac{\Lambda_{\tau}}{e^{4}}\right)^{1 / 3} .
$$

(In reality, the divergence is cut once we exit the two patch regime where the momentum $l_{x} \ll l_{y}$. This occurs when $l_{x}$ $\sim l_{\tau}^{2 / 3}$ becomes of order $l_{y}$. However, for the AslamazovLarkin diagrams the internal momentum $l_{y}$ is controlled by external momentum $q_{y}$. Hence, $\Lambda_{\tau} \sim q_{y}^{3 / 2}$ and $\delta^{3 a} \Pi=-\delta^{3 b} \Pi$ $\sim q_{y}^{3 / 2}$, as found in Ref. 26.)

However, as expected for problems involving a boson field coupled to the charge sector of the Fermi surface, the divergence cancels when we add the two diagrams. In fact, for $N \gg 1$, the divergence is cutoff at $\frac{c_{f}}{N} l_{\tau}^{2 / 3} \sim q_{y}^{2}$, i.e.,

$$
l_{\tau} \sim N^{3 / 2} q_{y}^{3} / e^{2}
$$

so that

$$
\delta^{3} \Pi(0, \vec{q}) \sim-\lambda_{+} \lambda_{-} N^{3 / 2} \frac{q_{y}^{2}}{e^{2}} .
$$

Note that the result is parametrically larger in the large- $N$ limit than the bare boson polarization, Eq. (2.7) (although it has the same scaling as the bare term). Also observe that the sign of the contribution, Eq. (B9), is positive for the spin liquid and negative for the Ising-nematic transition.

One may ask whether the enhancement in Eq. (B9) is an artifact of taking $q_{\tau}=0$. However, since the integral in Eq. (B9) is saturated in the region, Eq. (B8), we expect the result (B9) to be valid for, $q_{\tau} \ll N^{3 / 2} q_{y}^{3} / e^{2}$, which is certainly satisfied by the typical bosonic momenta $q_{\tau} \sim q_{y}^{3} / e^{2}$.

We can compute the proportionality factor in Eq. (B9) in the large- $N$ limit. Changing variables to $l_{\tau}=\left(\frac{N}{c_{f}}\right)^{3 / 2}\left|q_{y}\right|^{3} \bar{l}_{\tau}, p_{\tau}$ $=l_{\tau} x, p_{\tau}^{\prime}=l_{\tau} x^{\prime}, l_{y}=\left|q_{y}\right| y$,

$$
\delta^{3} \Pi(0, \vec{q})=C \lambda_{+} \lambda_{-} \frac{q_{y}^{2}}{e^{2}},
$$

$$
\begin{aligned}
C= & -\frac{2^{5 / 2} 3^{3 / 4} N^{3 / 2}}{\pi} \int_{0}^{\infty} d \bar{l}_{\tau} \int_{0}^{1} d x \int_{0}^{1} d x^{\prime} \int_{0}^{1} d y \\
& \times \frac{\bar{l}_{\tau}^{4 / 3} y^{3}(1-y)^{3}}{\left.\bar{l}_{\tau}+\left(\frac{2}{N \sqrt{3}}\right)^{3 / 2} y^{3}\right]\left[\bar{l}_{\tau}+\left(\frac{2}{N \sqrt{3}}\right)^{3 / 2}(1-y)^{3}\right]} \\
& \times \frac{1}{A\left[A^{2} \bar{l}_{\tau}^{4 / 3}+4 y^{2}(1-y)^{2}\right]}
\end{aligned}
$$

with,

$$
A=x^{2 / 3}+(1-x)^{2 / 3}+x^{\prime 2 / 3}+\left(1-x^{\prime}\right)^{2 / 3} .
$$

For $N \gg 1$, the integral over $\bar{l}_{\tau}$ is saturated in the region $\bar{l}_{\tau}$ $\sim 1$, so

$$
\begin{aligned}
C \approx & -\frac{2^{5 / 2} 3^{3 / 4} N^{3 / 2}}{\pi} \int_{0}^{\infty} \frac{d \bar{l}_{\tau}}{\bar{l}_{\tau}^{2 / 3}} \int_{0}^{1} d x \int_{0}^{1} d x^{\prime} \int_{0}^{1} d y \\
& \times \frac{y^{3}(1-y)^{3}}{A\left[A^{2} \bar{l}_{\tau}^{4 / 3}+4 y^{2}(1-y)^{2}\right]} .
\end{aligned}
$$

After a change of variables, $z=A \vec{l}_{\tau}^{2 / 3} /[2 y(1-y)]$,

$$
\begin{aligned}
C \approx & -\frac{3^{7 / 4} N^{3 / 2}}{\pi} \int_{0}^{\infty} \frac{d z}{z^{1 / 2}\left(z^{2}+1\right)} \int_{0}^{1} d y y^{3 / 2}(1-y)^{3 / 2} \\
& \times \int_{0}^{1} d x \int_{0}^{1} d x^{\prime} \frac{1}{A^{3 / 2}} \\
= & \frac{3^{11 / 4} \pi N^{3 / 2}}{2^{15 / 2}} \int_{0}^{1} d x \int_{0}^{1} d x^{\prime} \frac{1}{A^{3 / 2}} .
\end{aligned}
$$

The integral over $x, x^{\prime}$ can be evaluated numerically,

$$
\int_{0}^{1} d x \int_{0}^{1} d x^{\prime} \frac{1}{A^{3 / 2}}=0.269653
$$

so that

$$
C \approx-0.09601 N^{3 / 2}, \quad N \rightarrow \infty .
$$

We may also compute the constant $C$ in Eq. (B10) for the physical value $N=2$,

$$
C \approx-0.04455 .
$$




\section{Fermion self-energy}

We next compute the three-loop corrections to the fermion self-energy in diagrams in Figs. 10(b) and 10(c),

$$
\begin{aligned}
\delta^{3 b} \sum\left(p_{\tau}=\right. & 0, \vec{p})=N \lambda_{+}^{3} \lambda_{-}^{3} \int \frac{d k_{\tau} d^{2} k}{(2 \pi)^{3}} \frac{d l_{1 \tau} d^{2} l_{1}}{(2 \pi)^{3}} \frac{d l_{2 \tau} d^{2} l_{2}}{(2 \pi)^{3}} \\
& \times G_{+}\left(p-l_{1}\right) G_{+}\left(p-l_{2}\right) G_{-}(k) G_{-}\left(k+l_{1}\right) G_{-}\left(k+l_{2}\right) \\
& \times D\left(l_{1}\right) D\left(l_{2}\right) D\left(l_{1}-l_{2}\right),
\end{aligned}
$$

$$
\begin{aligned}
\delta^{3 c} \sum\left(p_{\tau}=\right. & 0, \vec{p})=N \lambda_{+}^{3} \lambda_{-}^{3} \int \frac{d k_{\tau} d^{2} k}{(2 \pi)^{3}} \frac{d l_{1 \tau} d^{2} l_{1}}{(2 \pi)^{3}} \frac{d l_{2 \tau} d^{2} l_{2}}{(2 \pi)^{3}} \\
& \times G_{+}\left(p+l_{1}\right) G_{+}\left(p+l_{2}\right) G_{-}(k) G_{-}\left(k+l_{1}\right) G_{-}\left(k+l_{2}\right) \\
& \times D\left(l_{1}\right) D\left(l_{2}\right) D\left(l_{1}-l_{2}\right) .
\end{aligned}
$$

$$
\begin{aligned}
& \delta^{3 b} \Sigma\left(p_{\tau}=0, \vec{p}\right)=-N \lambda_{+} \lambda_{-} \int \frac{d k_{\tau} d^{2} k}{(2 \pi)^{3}} \frac{d l_{1 \tau} d l_{1 y}}{(2 \pi)^{2}} \frac{d l_{2 \tau} d l_{2 y}}{(2 \pi)^{2}} D\left(l_{1}\right) D\left(l_{2}\right) D\left(l_{1}-l_{2}\right) \\
& \times \frac{1}{-\frac{i c_{f}}{N} k_{\tau}^{2 / 3}+\delta_{k}^{-}-\frac{i c_{f}}{N}\left[\left(l_{1}+k\right)_{\tau}^{2 / 3}+l_{1 \tau}^{2 / 3}\right]+\delta_{k}^{-}+2(k+p)_{y} l_{1 y}-\delta_{p}^{+}-\frac{i c_{f}}{N}\left[\left(l_{2}+k\right)_{\tau}^{2 / 3}+l_{2 \tau}^{2 / 3}\right]+\delta_{k}^{-}+2(k+p)_{y} l_{2 y}-\delta_{p}^{+}},
\end{aligned}
$$

$$
\begin{aligned}
\delta^{3 c} \Sigma\left(p_{\tau}=\right. & 0, \vec{p}) \\
= & -N \lambda_{+} \lambda_{-} \int \frac{d k_{\tau} d^{2} k}{(2 \pi)^{3}} \frac{d l_{1 \tau} d l_{1 y} \frac{d l_{2 \tau} d l_{2 y}}{(2 \pi)^{2}} D\left(l_{1}\right) D\left(l_{2}\right) D\left(l_{1}-l_{2}\right)}{(2 \pi)} \frac{\theta\left(l_{1 \tau}+k_{\tau}\right)-\theta\left(-l_{1 \tau}\right)}{} \times \frac{1}{-\frac{i c_{f}}{N} k_{\tau}^{2 / 3}+\delta_{k}^{-}-\frac{i c_{f}}{N}\left[\left(l_{1}+k\right)_{\tau}^{2 / 3}+l_{1 \tau}^{2 / 3}\right]+\delta_{k}+2(k+p)_{y} l_{1 y}+2 l_{1 y}^{2}+\delta_{p}^{+}} \\
& \times \frac{\theta\left(l_{2 \tau}+k_{\tau}\right)-\theta\left(-l_{2 \tau}\right)}{-\frac{i c_{f}}{N}\left[\left(l_{2}+k\right)_{\tau}^{2 / 3}+l_{2 \tau}^{2 / 3}\right]+\delta_{k}^{-}+2(k+p)_{y} l_{2 y}+2 l_{2 y}^{2}+\delta_{p}^{+}},
\end{aligned}
$$

where $\delta_{p}^{ \pm}= \pm p_{x}+p_{y}^{2}$. Note the cancellation of the Fermi-surface curvature terms $l_{1 y, 2 y}^{2}$ in the "planar graph" $\delta^{3 b \Sigma}$.

We can reduce the integration range to $k_{\tau}>0$ as the region $k_{\tau}<0$ is related by complex conjugation. There are then four different kinematic regimes: (i) $l_{1 \tau}>0, l_{2 \tau}>0$, (ii) $l_{1 \tau}<-k_{\tau}, l_{2 \tau}>0$, (iii) $l_{1 \tau}>0, l_{2 \tau}<-k_{\tau}$, and (iv) $l_{1 \tau}<-k_{\tau}, l_{2 \tau}<-k_{\tau}$. The integral over $k_{x}$ in the regime (i) vanishes as all the poles are in the same half plane. The regimes (ii) and (iii) are related by $l_{1} \leftrightarrow l_{2}$. Thus,

$$
\begin{aligned}
\delta^{3 b} \Sigma\left(p_{\tau}=\right. & 0, \vec{p}) \\
= & -N \lambda_{+} \lambda_{-}\left[-2 \int_{0}^{\infty} \frac{d k_{\tau}}{2 \pi} \int \frac{d^{2} k}{(2 \pi)^{2}} \int_{k_{\tau}}^{\infty} \frac{d l_{1 \tau}}{2 \pi} \int_{0}^{\infty} \frac{d l_{2 \tau}}{2 \pi} \int \frac{d l_{1 y} d l_{2 y}}{(2 \pi)^{2}} D\left(l_{1}\right) D\left(l_{2}\right) D\left(l_{1 \tau}+l_{2 \tau} l_{1 y}-l_{2 y}\right) \frac{1}{-\frac{i c_{f}}{N} k_{\tau}^{2 / 3}+\delta_{k}}\right. \\
& \times \frac{1}{\frac{i c_{f}}{N}\left(\left(l_{1}-k\right)_{\tau}^{2 / 3}+l_{1 \tau}^{2 / 3}\right)+\delta_{k}^{-}+2(k+p)_{y} l_{1 y}-\delta_{p}^{+}-\frac{i c_{f}}{N}\left(\left(l_{2}+k\right)_{\tau}^{2 / 3}+l_{2 \tau}^{2 / 3}\right)+\delta_{k}^{-}+2(k+p)_{y} l_{2 y}-\delta_{p}^{+}} \\
& +\int_{0}^{\infty} \frac{d k_{\tau}}{2 \pi} \int \frac{d^{2} k}{(2 \pi)^{2}} \int_{k_{\tau}}^{\infty} \frac{d l_{1 \tau}}{2 \pi} \int_{k_{\tau}}^{\infty} \frac{d l_{2 \tau}}{2 \pi} \int \frac{d l_{1 y} d l_{2 y}}{(2 \pi)^{2}} D\left(l_{1}\right) D\left(l_{2}\right) D\left(l_{1}-l_{2}\right) \\
& \left.\times \frac{1}{-\frac{i c_{f}}{N} k_{\tau}^{2 / 3}+\delta_{k}^{-} \frac{i c_{f}}{N}\left(\left(l_{1}-k\right)_{\tau}^{2 / 3}+l_{1 \tau}^{2 / 3}\right)+\delta_{k}^{-}+2(k+p)_{y} l_{1 y}-\delta_{p}^{+} \frac{i c_{f}}{N}\left(\left(l_{2}-k\right)_{\tau}^{2 / 3}+l_{2 \tau}^{2 / 3}\right)+\delta_{k}^{-}+2(k+p)_{y} l_{2 y}-\delta_{p}^{+}}\right]+ \text {H.c. },
\end{aligned}
$$




$$
\begin{aligned}
\delta^{3 c \Sigma}\left(p_{\tau}=\right. & 0, \vec{p}) \\
= & -N \lambda_{+} \lambda_{-}\left\{-2 \int_{0}^{\infty} \frac{d k_{\tau}}{2 \pi} \int \frac{d^{2} k}{(2 \pi)^{2}} \int_{k_{\tau}}^{\infty} \frac{d l_{1 \tau}}{2 \pi} \int_{0}^{\infty} \frac{d l_{2 \tau}}{2 \pi} \int \frac{d l_{1 y} d l_{2 y}}{(2 \pi)^{2}} D\left(l_{1}\right) D\left(l_{2}\right) D\left(l_{1 \tau}+l_{2 \tau}, l_{1 y}-l_{2 y}\right) \frac{1}{i c_{f} k_{\tau}^{2 / 3}+\delta_{k}^{-}}\right. \\
& \times \frac{1}{\frac{i c_{f}}{N}\left(\left(l_{1}-k\right)_{\tau}^{2 / 3}+l_{1 \tau}^{2 / 3}\right)+\delta_{k}^{-}+2(k+p)_{y} l_{1 y}+2 l_{1 y}^{2}+\delta_{p}^{+}-\frac{i c_{f}}{N}\left[\left(l_{2}+k\right)_{\tau}^{2 / 3}+l_{2 \tau}^{2 / 3}\right]+\delta_{k}+2(k+p)_{y} l_{2 y}+2 l_{2 y}^{2}+\delta_{p}^{+}} \\
& +\int_{0}^{\infty} \frac{d k_{\tau}}{2 \pi} \int \frac{d^{2} k}{(2 \pi)^{2}} \int_{k_{\tau}}^{\infty} \frac{d l_{1 \tau}}{2 \pi} \int_{k_{\tau}}^{\infty} \frac{d l_{2 \tau}}{2 \pi} \int \frac{d l_{1 y} d l_{2 y}}{(2 \pi)^{2}} D\left(l_{1}\right) D\left(l_{2}\right) D\left(l_{1}-l_{2}\right) \frac{1}{-\frac{i c_{f}}{N} k_{\tau}^{2 / 3}+\delta_{k}} \\
& \left.\times \frac{1}{\frac{i c_{f}}{N}\left(\left(l_{1}-k\right)_{\tau}^{2 / 3}+l_{1 \tau}^{2 / 3}\right)+\delta_{k}^{-}+2(k+p)_{y} l_{1 y}+2 l_{1 y}^{2}+\delta_{p}^{+} \frac{i c_{f}}{N}\left[\left(l_{2}-k\right)_{\tau}^{2 / 3}+l_{2 \tau}^{2 / 3}\right]+\delta_{k}^{-}+2(k+p)_{y} l_{2 y}+2 l_{2 y}^{2}+\delta_{p}^{+}}\right\}+\mathrm{H.c.}
\end{aligned}
$$

Integrating over $k_{x}$ and shifting $k_{y} \rightarrow k_{y}-p$,

$$
\begin{aligned}
& \delta^{3 b} \Sigma\left(p_{\tau}=0, \vec{p}\right)=-N \lambda_{+} \lambda_{-}\left\{2 i \int_{0}^{\infty} \frac{d k_{\tau}}{2 \pi} \int \frac{d k_{y}}{2 \pi} \int_{k_{\tau}}^{\infty} \frac{d l_{1 \tau}}{2 \pi} \int_{0}^{\infty} \frac{d l_{2 \tau}}{2 \pi} \int \frac{d l_{1 y} d l_{2 y}}{(2 \pi)^{2}} D\left(l_{1}\right) D\left(l_{2}\right) D\left(l_{1 \tau}+l_{2 \tau}, l_{1 y}-l_{2 y}\right)\right. \\
& \times \frac{1}{-\frac{i c_{f}}{N}\left[k_{\tau}^{2 / 3}+\left(l_{1}-k\right)_{\tau}^{2 / 3}+l_{1 \tau}^{2 / 3}\right]-2 k_{y} l_{1 y}+\delta_{p}^{+}-\frac{i c_{f}}{N}\left[\left(l_{1}-k\right)_{\tau}^{2 / 3}+l_{1 \tau}^{2 / 3}+\left(l_{2}+k\right)_{\tau}^{2 / 3}+l_{2 \tau}^{2 / 3}\right]+2 k_{y}\left(l_{2}-l_{1}\right)_{y}} \\
& +i \int_{0}^{\infty} \frac{d k_{\tau}}{2 \pi} \int \frac{d k_{y}}{2 \pi} \int_{k_{\tau}}^{\infty} \frac{d l_{1 \tau}}{2 \pi} \int_{k_{\tau}}^{\infty} \frac{d l_{2 \tau}}{2 \pi} \int \frac{d l_{1 y} d l_{2 y}}{(2 \pi)^{2}} D\left(l_{1}\right) D\left(l_{2}\right) D\left(l_{1}-l_{2}\right) \\
& \left.\times \frac{1}{\frac{i c_{f}}{N}\left[k_{\tau}^{2 / 3}+\left(l_{1}-k\right)_{\tau}^{2 / 3}+l_{1 \tau}^{2 / 3}\right]+2 k_{y} l_{1 y}-\delta_{p}^{+} \frac{i c_{f}}{N}\left[k_{\tau}^{2 / 3}+\left(l_{2}-k\right)_{\tau}^{2 / 3}+l_{2 \tau}^{2 / 3}\right]+2 k_{y} l_{2 y}-\delta_{p}^{+}}\right\}+ \text {H.c., } \\
& \delta^{3 c} \Sigma\left(p_{\tau}=0, \vec{p}\right)=-N \lambda_{+} \lambda_{-}\left\{2 i \int_{0}^{\infty} \frac{d k_{\tau}}{2 \pi} \int \frac{d k_{y}}{2 \pi} \int_{k_{\tau}}^{\infty} \frac{d l_{1 \tau}}{2 \pi} \int_{0}^{\infty} \frac{d l_{2 \tau}}{2 \pi} \int \frac{d l_{1 y} d l_{2 y}}{(2 \pi)^{2}} D\left(l_{1}\right) D\left(l_{2}\right) D\left(l_{1 \tau}+l_{2 \tau}, l_{1 y}-l_{2 y}\right)\right. \\
& \times \frac{1}{-\frac{i c_{f}}{N}\left[k_{\tau}^{2 / 3}+\left(l_{1}-k\right)_{\tau}^{2 / 3}+l_{1 \tau}^{2 / 3}\right]-2 k_{y} l_{1 y}-2 l_{1 y}^{2}-\delta_{p}^{+}-\frac{i c_{f}}{N}\left[\left(l_{1}-k\right)_{\tau}^{2 / 3}+l_{1 \tau}^{2 / 3}+\left(l_{2}+k\right)_{\tau}^{2 / 3}+l_{2 \tau}^{2 / 3}\right]+2 k_{y}\left(l_{2}-l_{1}\right)_{y}+2\left(l_{2 y}^{2}-l_{1 y}^{2}\right)} \\
& +i \int_{0}^{\infty} \frac{d k_{\tau}}{2 \pi} \int \frac{d k_{y}}{2 \pi} \int_{k_{\tau}}^{\infty} \frac{d l_{1 \tau}}{2 \pi} \int_{k_{\tau}}^{\infty} \frac{d l_{2 \tau}}{2 \pi} \int \frac{d l_{1 y} d l_{2 y}}{(2 \pi)^{2}} D\left(l_{1}\right) D\left(l_{2}\right) D\left(l_{1}-l_{2}\right) \\
& \left.\times \frac{1}{\frac{i c_{f}}{N}\left[k_{\tau}^{2 / 3}+\left(l_{1}-k\right)_{\tau}^{2 / 3}+l_{1 \tau}^{2 / 3}\right]+2 k_{y} l_{1 y}+2 l_{1 y}^{2}+\delta_{p}^{+} \frac{i c_{f}}{N}\left[k_{\tau}^{2 / 3}+\left(l_{2}-k\right)_{\tau}^{2 / 3}+l_{2 \tau}^{2 / 3}\right]+2 k_{y} l_{2 y}+2 l_{2 y}^{2}+\delta_{p}^{+}}\right\}+ \text {H.c. }
\end{aligned}
$$

The integration regions $l_{1 y}>0$ and $l_{1 y}<0$ give the same contribution. So, integrating over $k_{y}$, 


$$
\begin{aligned}
& \delta^{3 b} \Sigma\left(p_{\tau}=0, \vec{p}\right)=N \lambda_{+} \lambda_{-}\left[2 \int_{0}^{\infty} \frac{d k_{\tau}}{2 \pi} \int_{k_{\tau}}^{\infty} \frac{d l_{1 \tau}}{2 \pi} \int_{0}^{\infty} \frac{d l_{2 \tau}}{2 \pi} \int_{0}^{\infty} \frac{d l_{1 y}}{2 \pi} \int_{l_{1 y}}^{\infty} \frac{d l_{2 y}}{2 \pi} D\left(l_{1}\right) D\left(l_{2}\right) D\left(l_{1 \tau}+l_{2 \tau}, l_{1 y}-l_{2 y}\right)\right. \\
& \times \frac{1}{-\frac{i c_{f}}{N}\left\{l_{2 y}\left[\left(l_{1}-k\right)_{\tau}^{2 / 3}+l_{1 \tau}^{2 / 3}+k_{\tau}^{2 / 3}\right]+l_{1 y}\left[\left(l_{2}+k\right)_{\tau}^{2 / 3}+l_{2 \tau}^{2 / 3}-k_{\tau}^{2 / 3}\right]\right\}+\left(l_{2}-l_{1}\right)_{y} \delta_{p}^{+}} \\
& +\int_{0}^{\infty} \frac{d k_{\tau}}{2 \pi} \int_{k_{\tau}}^{\infty} \frac{d l_{1 \tau}}{2 \pi} \int_{k_{\tau}}^{\infty} \frac{d l_{2 \tau}}{2 \pi} \int_{0}^{\infty} \frac{d l_{1 y}}{2 \pi} \int_{0}^{\infty} \frac{d l_{2 y}}{2 \pi} D\left(l_{1}\right) D\left(l_{2}\right) D\left(l_{1 \tau}-l_{2 \tau}, l_{1 y}+l_{2 y}\right) \\
& \left.\frac{1}{-\frac{i c_{f}}{N}\left\{l_{2 y}\left[\left(l_{1}-k\right)_{\tau}^{2 / 3}+l_{1 \tau}^{2 / 3}+k_{\tau}^{2 / 3}\right]+l_{1 y}\left[\left(l_{2}-k\right)_{\tau}^{2 / 3}+l_{2 \tau}^{2 / 3}+k_{\tau}^{2 / 3}\right]\right\}+\left(l_{1}+l_{2}\right)_{y} \delta_{p}^{+}}\right]+ \text {H.c., } \\
& \delta^{3 c} \Sigma\left(p_{\tau}=0, \vec{p}\right)=N \lambda_{+} \lambda_{-}\left[2 \int_{0}^{\infty} \frac{d k_{\tau}}{2 \pi} \int_{k_{\tau}}^{\infty} \frac{d l_{1 \tau}}{2 \pi} \int_{0}^{\infty} \frac{d l_{2 \tau}}{2 \pi} \int_{0}^{\infty} \frac{d l_{1 y}}{2 \pi} \int_{l_{1 y}}^{\infty} \frac{d l_{2 y}}{2 \pi} D\left(l_{1}\right) D\left(l_{2}\right) D\left(l_{1 \tau}+l_{2 \tau}, l_{1 y}-l_{2 y}\right)\right. \\
& \times \frac{1}{-\frac{i c_{f}}{N}\left\{l_{2 y}\left[\left(l_{1}-k\right)_{\tau}^{2 / 3}+l_{1 \tau}^{2 / 3}+k_{\tau}^{2 / 3}\right]+l_{1 y}\left[\left(l_{2}+k\right)_{\tau}^{2 / 3}+l_{2 \tau}^{2 / 3}-k_{\tau}^{2 / 3}\right]\right\}+2 l_{1 y} l_{2 y}\left(l_{2}-l_{1}\right)_{y}-\left(l_{2}-l_{1}\right)_{y} \delta_{p}^{+}} \\
& +\int_{0}^{\infty} \frac{d k_{\tau}}{2 \pi} \int_{k_{\tau}}^{\infty} \frac{d l_{1 \tau}}{2 \pi} \int_{k_{\tau}}^{\infty} \frac{d l_{2 \tau}}{2 \pi} \int_{0}^{\infty} \frac{d l_{1 y}}{2 \pi} \int_{0}^{\infty} \frac{d l_{2 y}}{2 \pi} D\left(l_{1}\right) D\left(l_{2}\right) D\left(l_{1 \tau}-l_{2 \tau}, l_{1 y}+l_{2 y}\right) \\
& \left.\times \frac{1}{-\frac{i c_{f}}{N}\left\{l_{2 y}\left[\left(l_{1}-k\right)_{\tau}^{2 / 3}+l_{1 \tau}^{2 / 3}+k_{\tau}^{2 / 3}\right]+l_{1 y}\left[\left(l_{2}-k\right)_{\tau}^{2 / 3}+l_{2 \tau}^{2 / 3}+k_{\tau}^{2 / 3}\right]\right\}-2 l_{1 y} l_{2 y}\left(l_{1}+l_{2}\right)_{y}-\left(l_{1}+l_{2}\right)_{y} \delta_{p}^{+}}\right]+ \text {H.c. }
\end{aligned}
$$

Expanding the self-energy in $\delta_{p}^{+}$and performing a change of variables $l_{1 \tau}=k_{\tau} x_{1}, l_{2 \tau}=k_{\tau} x_{2}, l_{1 y}=\left(c_{b} e^{2} k_{\tau}\right)^{1 / 3} y_{1}, l_{2 y}=\left(c_{b} e^{2} k_{\tau}\right)^{1 / 3} y_{2}$,

$$
\begin{gathered}
\delta^{3 b} \Sigma_{+}\left(p_{\tau}=0, \vec{p}\right)=\lambda_{+} \lambda_{-}\left(J_{1}+J_{2}\right) \delta_{p}^{+} \int_{0}^{\infty} \frac{d k_{\tau}}{k_{\tau}}, \\
\delta^{3 c \Sigma_{+}}\left(p_{\tau}=0, \vec{p}\right)=\delta^{3 c} \Sigma_{+}\left(p_{\tau}=0, \vec{p}=0\right)+\lambda_{+} \lambda_{-}\left(J_{3}+J_{4}\right) \delta_{p}^{+} \int_{0}^{\infty} \frac{d k_{\tau}}{k_{\tau}},
\end{gathered}
$$

where

$$
\begin{aligned}
J_{1}= & \frac{6}{\pi^{2}} \int_{1}^{\infty} d x_{1} \int_{0}^{\infty} d x_{2} \int_{0}^{\infty} d y_{1} \int_{y_{1}}^{\infty} d y_{2} \frac{y_{1} y_{2}\left(y_{2}-y_{1}\right)^{2}}{\left(x_{1}+y_{1}^{3}\right)\left(x_{2}+y_{2}^{3}\right)\left[x_{1}+x_{2}+\left(y_{2}-y_{1}\right)^{3}\right]} \\
& \times \frac{1}{\left\{y_{2}\left[\left(x_{1}-1\right)^{2 / 3}+x_{1}^{2 / 3}+1\right]+y_{1}\left[\left(x_{2}+1\right)^{2 / 3}+x_{2}^{2 / 3}-1\right]\right\}^{2}}, \\
J_{2}= & \frac{3}{\pi^{2}} \int_{1}^{\infty} d x_{1} \int_{1}^{\infty} d x_{2} \int_{0}^{\infty} d y_{1} \int_{0}^{\infty} d y_{2} \frac{y_{1} y_{2}\left(y_{1}+y_{2}\right)^{2}}{\left(x_{1}+y_{1}^{3}\right)\left(x_{2}+y_{2}^{3}\right)\left[\left|x_{1}-x_{2}\right|+\left(y_{1}+y_{2}\right)^{3}\right]} \\
& \times \frac{1}{\left\{y_{2}\left[\left(x_{1}-1\right)^{2 / 3}+x_{1}^{2 / 3}+1\right]+y_{1}\left[\left(x_{2}-1\right)^{2 / 3}+x_{2}^{2 / 3}+1\right]\right\}^{2}},
\end{aligned}
$$




$$
\begin{aligned}
J_{3}= & \frac{6}{\pi^{2} N^{2}} \int_{1}^{\infty} d x_{1} \int_{0}^{\infty} d x_{2} \int_{0}^{\infty} d y_{1} \int_{y_{1}}^{\infty} d y_{2} \frac{y_{1} y_{2}\left(y_{2}-y_{1}\right)^{2}}{\left(x_{1}+y_{1}^{3}\right)\left(x_{2}+y_{2}^{3}\right)\left[x_{1}+x_{2}+\left(y_{2}-y_{1}\right)^{3}\right]} \\
& \times \frac{3 y_{1}^{2} y_{2}^{2}\left(y_{2}-y_{1}\right)^{2}-\frac{1}{N^{2}}\left\{y_{2}\left[\left(x_{1}-1\right)^{2 / 3}+x_{1}^{2 / 3}+1\right]+y_{1}\left[\left(x_{2}+1\right)^{2 / 3}+x_{2}^{2 / 3}-1\right]\right\}^{2}}{\left(3 y_{1}^{2} y_{2}^{2}\left(y_{2}-y_{1}\right)^{2}+\frac{1}{N^{2}}\left\{y_{2}\left[\left(x_{1}-1\right)^{2 / 3}+x_{1}^{2 / 3}+1\right]+y_{1}\left[\left(x_{2}+1\right)^{2 / 3}+x_{2}^{2 / 3}-1\right]\right\}^{2}\right)^{2}}, \\
J_{4}= & \frac{3}{\pi^{2} N^{2}} \int_{1}^{\infty} d x_{1} \int_{1}^{\infty} d x_{2} \int_{0}^{\infty} d y_{1} \int_{0}^{\infty} d y_{2} \frac{y_{1} y_{2}\left(y_{1}+y_{2}\right)^{2}}{\left(x_{1}+y_{1}^{3}\right)\left(x_{2}+y_{2}^{3}\right)\left[\left|x_{1}-x_{2}\right|+\left(y_{1}+y_{2}\right)^{3}\right]} \\
& \times \frac{3 y_{1}^{2} y_{2}^{2}\left(y_{1}+y_{2}\right)^{2}-\frac{1}{N^{2}}\left\{y_{2}\left[\left(x_{1}-1\right)^{2 / 3}+x_{1}^{2 / 3}+1\right]+y_{1}\left[\left(x_{2}-1\right)^{2 / 3}+x_{2}^{2 / 3}+1\right]\right\}^{2}}{\left(3 y_{1}^{2} y_{2}^{2}\left(y_{1}+y_{2}\right)^{2}+\frac{1}{N^{2}}\left\{y_{2}\left[\left(x_{1}-1\right)^{2 / 3}+x_{1}^{2 / 3}+1\right]+y_{1}\left[\left(x_{2}-1\right)^{2 / 3}+x_{2}^{2 / 3}+1\right]\right\}^{2}\right)^{2}} .
\end{aligned}
$$

Cutting off the UV divergence in Eqs. (B28) and (B29) at $k_{\tau}=\Lambda_{\tau} \sim \Lambda_{y}^{3} / e^{2}$, we obtain to logarithmic accuracy,

$$
\begin{aligned}
& \delta^{3 b} \Sigma_{+}\left(p_{\tau}=0, \vec{p}\right)=\lambda_{+} \lambda_{-}\left(J_{1}+J_{2}\right) \delta_{p}^{+} \log \frac{\Lambda_{y}^{3}}{\left|\delta_{p}^{+}\right|^{3 / 2}}, \\
& \delta^{3 c} \Sigma_{+}\left(p_{\tau}=0, \vec{p}\right)=\delta^{3 c} \Sigma_{+}\left(p_{\tau}=0, \vec{p}=0\right) \\
& +\lambda_{+} \lambda_{-}\left(J_{3}+J_{4}\right) \delta_{p}^{+} \log \frac{\Lambda_{y}^{3}}{\left|\delta_{p}^{+}\right|^{3 / 2}},
\end{aligned}
$$

which is equivalent to Eqs. (5.9) and (5.10) with $J_{b}=3$ $\left(J_{1}+J_{2}\right)$ and $J_{c}=3\left(J_{3}+J_{4}\right)$. Note that $J_{1}$ and $J_{2}$ are constants independent of $N$,

$$
\begin{aligned}
& J_{1} \approx 0.01276, \\
& J_{2} \approx 0.02264 .
\end{aligned}
$$

On the other hand, the constants $J_{3}$ and $J_{4}$ are $N$ dependent. In the large- $N$ limit we can evaluate these constants analytically to leading logarithmic accuracy by setting $N=\infty$ in the integrand,

$$
\begin{aligned}
J_{3} \approx & \frac{2}{\pi^{2} N^{2}} \int_{1}^{\infty} d x_{1} \int_{0}^{\infty} d x_{2} \int_{0}^{\infty} d y_{1} \int_{y_{1}}^{\infty} d y_{2} \\
& \times \frac{1}{y_{1} y_{2}\left(x_{1}+y_{1}^{3}\right)\left(x_{2}+y_{2}^{3}\right)\left(x_{1}+x_{2}+\left(y_{2}-y_{1}\right)^{3}\right)} .
\end{aligned}
$$

The above integral diverges logarithmically when $y_{1}, y_{2}, x_{2}$ $\rightarrow 0$. Hence,

$$
\begin{aligned}
J_{3} & \approx \frac{2}{\pi^{2} N^{2}} \int_{1}^{\infty} \frac{d x_{1}}{x_{1}^{2}} \int_{0}^{1} d x_{2} \int_{0}^{1} d y_{1} \int_{y_{1}}^{1} d y_{2} \frac{1}{y_{1} y_{2}\left(x_{2}+y_{2}^{3}\right)} \\
& \approx \frac{2}{\pi^{2} N^{2}} \int_{0}^{1} \frac{d y_{2}}{y_{2}} \log \left(y_{2}^{-3}\right) \int_{0}^{y_{2}} \frac{d y_{1}}{y_{1}}
\end{aligned}
$$

Inspecting the original integral, Eq. (B32), we observe that the logarithmic divergence in Eq. (B39) is cutoff when $y_{1}\left(y_{2}-y_{1}\right) \sim \frac{1}{N}$. Hence,

$$
J_{3} \approx \frac{2}{\pi^{2} N^{2}} \int_{N^{-1 / 2}}^{1} \frac{d y_{2}}{y_{2}} \log \left(y_{2}^{-3}\right) \int_{\left(N y_{2}\right)^{-1}}^{y_{2}} \frac{d y_{1}}{y_{1}} \approx \frac{1}{4 \pi^{2} N^{2}} \log ^{3} N .
$$

Similarly,

$$
\begin{aligned}
J_{4} \approx & \frac{1}{\pi^{2} N^{2}} \int_{1}^{\infty} d x_{1} \int_{1}^{\infty} d x_{2} \int_{0}^{\infty} d y_{1} \int_{0}^{\infty} d y_{2} \\
& \times \frac{1}{y_{1} y_{2}\left(x_{1}+y_{1}^{3}\right)\left(x_{2}+y_{2}^{3}\right)\left[\left|x_{1}-x_{2}\right|+\left(y_{1}+y_{2}\right)^{3}\right]} \\
\approx & \frac{4}{\pi^{2} N^{2}} \int_{1}^{\infty} \frac{d x_{1}}{x_{1}^{2}} \int_{0}^{1} \frac{d y_{2}}{y_{2}} \int_{0}^{y_{2}} \frac{d y_{1}}{y_{1}} \log \left[\left(y_{1}+y_{2}\right)^{-3}\right] .
\end{aligned}
$$

Inspecting Eq. (B33), we see that the logarithmic divergence in Eq. (B41) is cutoff when $y_{1} y_{2} \sim \frac{1}{N}$. Writing, $y_{1}=y_{2} z$,

$$
\begin{aligned}
J_{4} & \approx-\frac{12}{\pi^{2} N^{2}} \int_{N^{-1 / 2}}^{1} \frac{d y_{2}}{y_{2}} \int_{\left(N y_{2}^{2}\right)^{-1}}^{1} \frac{d z}{z}\left[\log y_{2}+\log (1+z)\right] \\
& \approx \frac{1}{2 \pi^{2} N^{2}} \log ^{3} N
\end{aligned}
$$

We note that expressions (B40) and (B42) do not include subleading polynomial corrections in $\log N$. We can also calculate the constants $J_{3}, J_{4}$ numerically for $N=2$, 


$$
\begin{aligned}
& J_{3} \approx-0.004491, \\
& J_{4} \approx-0.008158 .
\end{aligned}
$$

Finally, we compute the insertion of the $\phi^{2}$ operator into the fermion two-point function, which determines the renor- malization of the chemical potential $\delta$ away from criticality. The UV contribution at three-loop order comes from the diagrams in Figs. 10(b) and 10(c) and can be obtained by expanding the bosonic propagators in Eqs. (B26) and (B27) to linear order in $r$. This yields,

$$
\begin{aligned}
& \delta^{3 b} \frac{\partial \Sigma^{\mathrm{UV}}}{\partial r}=-N\left[2 \int_{0}^{\infty} \frac{d k_{\tau}}{2 \pi} \int_{k_{\tau}}^{\infty} \frac{d l_{1 \tau}}{2 \pi} \int_{0}^{\infty} \frac{d l_{2 \tau}}{2 \pi} \int_{0}^{\infty} \frac{d l_{1 y}}{2 \pi} \int_{l_{1 y}}^{\infty} \frac{d l_{2 y}}{2 \pi}\left[D\left(l_{1}\right)+D\left(l_{2}\right)+D\left(l_{1 \tau}+l_{2 \tau}, l_{1 y}-l_{2 y}\right)\right] D\left(l_{1}\right) D\left(l_{2}\right) D\left(l_{1 \tau}+l_{2 \tau}, l_{1 y}-l_{2 y}\right)\right. \\
& \frac{1}{-\frac{i c_{f}}{N}\left\{l_{2 y}\left[\left(l_{1}-k\right)_{\tau}^{2 / 3}+l_{1 \tau}^{2 / 3}+k_{\tau}^{2 / 3}\right]+l_{1 y}\left[\left(l_{2}+k\right)_{\tau}^{2 / 3}+l_{2 \tau}^{2 / 3}-k_{\tau}^{2 / 3}\right]\right\}}+\int_{0}^{\infty} \frac{d k_{\tau}}{2 \pi} \int_{k_{\tau}}^{\infty} \frac{d l_{1 \tau}}{2 \pi} \int_{k_{\tau}}^{\infty} \frac{d l_{2 \tau}}{2 \pi} \int_{0}^{\infty} \frac{d l_{1 y}}{2 \pi} \int_{0}^{\infty} \frac{d l_{2 y}}{2 \pi} \\
& \times\left[D\left(l_{1}\right)+D\left(l_{2}\right)+D\left(l_{1 \tau}-l_{2 \tau}, l_{1 y}+l_{2 y}\right)\right] D\left(l_{1}\right) D\left(l_{2}\right) D\left(l_{1 \tau}-l_{2 \tau}, l_{1 y}+l_{2 y}\right) \\
& \left.\times \frac{1}{-\frac{i c_{f}}{N}\left\{l_{2 y}\left[\left(l_{1}-k\right)_{\tau}^{2 / 3}+l_{1 \tau}^{2 / 3}+k_{\tau}^{2 / 3}\right]+l_{1 y}\left[\left(l_{2}-k\right)_{\tau}^{2 / 3}+l_{2 \tau}^{2 / 3}+k_{\tau}^{2 / 3}\right]\right\}}\right]+ \text { H.c., } \\
& \delta^{3 c} \frac{\partial \Sigma^{\mathrm{UV}}}{\partial r}=-N\left[2 \int_{0}^{\infty} \frac{d k_{\tau}}{2 \pi} \int_{k_{\tau}}^{\infty} \frac{d l_{1 \tau}}{2 \pi} \int_{0}^{\infty} \frac{d l_{2 \tau}}{2 \pi} \int_{0}^{\infty} \frac{d l_{1 y}}{2 \pi} \int_{l_{1 y}}^{\infty} \frac{d l_{2 y}}{2 \pi}\left[D\left(l_{1}\right)+D\left(l_{2}\right)+D\left(l_{1 \tau}+l_{2 \tau}, l_{1 y}-l_{2 y}\right)\right] D\left(l_{1}\right) D\left(l_{2}\right) D\left(l_{1 \tau}+l_{2 \tau}, l_{1 y}-l_{2 y}\right)\right. \\
& \times \frac{1}{-\frac{i c_{f}}{N}\left\{l_{2 y}\left[\left(l_{1}-k\right)_{\tau}^{2 / 3}+l_{1 \tau}^{2 / 3}+k_{\tau}^{2 / 3}\right]+l_{1 y}\left[\left(l_{2}+k\right)_{\tau}^{2 / 3}+l_{2 \tau}^{2 / 3}-k_{\tau}^{2 / 3}\right]\right\}+2 l_{1 y} l_{2 y}\left(l_{2}-l_{1}\right)_{y}} \\
& +\int_{0}^{\infty} \frac{d k_{\tau}}{2 \pi} \int_{k_{\tau}}^{\infty} \frac{d l_{1 \tau}}{2 \pi} \int_{k_{\tau}}^{\infty} \frac{d l_{2 \tau}}{2 \pi} \int_{0}^{\infty} \frac{d l_{1 y}}{2 \pi} \int_{0}^{\infty} \frac{d l_{2 y}}{2 \pi}\left[D\left(l_{1}\right)+D\left(l_{2}\right)+D\left(l_{1 \tau}-l_{2 \tau}, l_{1 y}+l_{2 y}\right)\right] D\left(l_{1}\right) D\left(l_{2}\right) D\left(l_{1 \tau}-l_{2 \tau}, l_{1 y}+l_{2 y}\right) \\
& \left.\times \frac{1}{-\frac{i c_{f}}{N}\left\{l_{2 y}\left[\left(l_{1}-k\right)_{\tau}^{2 / 3}+l_{1 \tau}^{2 / 3}+k_{\tau}^{2 / 3}\right]+l_{1 y}\left[\left(l_{2}-k\right)_{\tau}^{2 / 3}+l_{2 \tau}^{2 / 3}+k_{\tau}^{2 / 3}\right]\right\}-2 l_{1 y} l_{2 y}\left(l_{1}+l_{2}\right)_{y}}\right]+ \text { H.c. }
\end{aligned}
$$

We observe that the contribution from the diagram in Fig. 10(b) vanishes while the diagram in Fig. 10(c) gives upon switching to dimensionless variables,

$$
\delta^{3} \frac{\partial \Sigma}{\partial r}=J_{r} e^{2} \log \Lambda_{y}
$$

with

$$
\begin{aligned}
J_{r}= & -\frac{36}{\pi^{2} N^{2}} \int_{1}^{\infty} d x_{1} \int_{0}^{\infty} d x_{2} \int_{0}^{\infty} d y_{1} \int_{y_{1}}^{\infty} d y_{2} \frac{y_{1}^{2} y_{2}^{2}\left(y_{2}-y_{1}\right)^{2}}{\left(x_{1}+y_{1}^{3}\right)\left(x_{2}+y_{2}^{3}\right)\left[x_{1}+x_{2}+\left(y_{2}-y_{1}\right)^{3}\right]}\left[\frac{y_{1}}{x_{1}+y_{1}^{3}}+\frac{y_{2}}{x_{2}+y_{2}^{3}}\right. \\
& \left.+\frac{y_{2}-y_{1}}{x_{1}+x_{2}+\left(y_{2}-y_{1}\right)^{3}}\right] \frac{1}{3 y_{1}^{2} y_{2}^{2}\left(y_{2}-y_{1}\right)^{2}+\frac{1}{N^{2}}\left\{y_{2}\left[\left(x_{1}-1\right)^{2 / 3}+x_{1}^{2 / 3}+1\right]+y_{1}\left[\left(x_{2}+1\right)^{2 / 3}+x_{2}^{2 / 3}-1\right]\right\}^{2}} \\
& +\frac{18}{\pi^{2} N^{2}} \int_{1}^{\infty} d x_{1} \int_{1}^{\infty} d x_{2} \int_{0}^{\infty} d y_{1} \int_{0}^{\infty} d y_{2} \frac{y_{1}^{2} y_{2}^{2}\left(y_{1}+y_{2}\right)^{2}}{\left(x_{1}+y_{1}^{3}\right)\left(x_{2}+y_{2}^{3}\right)\left[\left|x_{1}-x_{2}\right|+\left(y_{1}+y_{2}\right)^{3}\right]}\left[\frac{y_{1}}{x_{1}+y_{1}^{3}}+\frac{y_{2}}{x_{2}+y_{2}^{3}}\right.
\end{aligned}
$$




$$
\left.+\frac{y_{1}+y_{2}}{\left|x_{1}-x_{2}\right|+\left(y_{1}+y_{2}\right)^{3}}\right] \frac{1}{3 y_{1}^{2} y_{2}^{2}\left(y_{1}+y_{2}\right)^{2}+\frac{1}{N^{2}}\left\{y_{2}\left[\left(x_{1}-1\right)^{2 / 3}+x_{1}^{2 / 3}+1\right]+y_{1}\left[\left(x_{2}-1\right)^{2 / 3}+x_{2}^{2 / 3}+1\right]\right\}^{2}} .
$$

Evaluating the above integral, we obtain Eq. (5.18).

${ }^{1}$ Y. Ando, K. Segawa, S. Komiya, and A. N. Lavrov, Phys. Rev. Lett. 88, 137005 (2002).

${ }^{2}$ V. Hinkov, D. Haug, B. Fauqué, P. Bourges, Y. Sidis, A. Ivanov, C. Bernhard, C. T. Lin, and B. Keimer, Science 319, 597 (2008).

${ }^{3}$ Y. Kohsaka, C. Taylor, K. Fujita, A. Schmidt, C. Lupien, T. Hanaguri, M. Azuma, M. Takano, H. Eisaki, H. Takagi, S. Uchida, and J. C. Davis, Science 315, 1380 (2007).

${ }^{4}$ R. Daou, J. Chang, D. LeBoeuf, O. Cyr-Choiniere, F. Laliberte, N. Doiron-Leyraud, B. J. Ramshaw, R. Liang, D. A. Bonn, W. N. Hardy, and L. Taillefer, Nature (London) 463, 519 (2010).

${ }^{5}$ R. A. Borzi, S. A. Grigera, J. Ferrell, R. S. Perry, S. J. S. Lister, S. L. Lee, D. A. Tenant, Y. Maeno, and A. P. Mackenzie, Science 315, 214 (2007).

${ }^{6}$ C. Fang, H. Yao, W.-F. Tsai, J.-P. Hu, and S. A. Kivelson, Phys. Rev. B 77, 224509 (2008).

${ }^{7}$ C. Xu, M. Müller, and S. Sachdev, Phys. Rev. B 78, 020501(R) (2008).

${ }^{8}$ T.-M. Chuang, M. P. Allan, J. Lee, Y. Xie, N. Ni, S. L. Budko, G. S. Boebinger, P. C. Canfield, and J. C. Davis, Science 327, 181 (2010).

${ }^{9}$ J. Chu, J. Analytis, K. De Greve, P. McMahon, Z. Islam, Y. Yamamoto, and I. Fisher, arXiv:1002.3364 (unpublished).

${ }^{10}$ S. A. Kivelson, E. Fradkin, and V. J. Emery, Nature (London) 393, 550 (1998).

${ }^{11}$ J. Zaanen, J. Phys. Chem. Solids 59, 1769 (1998).

${ }^{12}$ S. A. Kivelson, I. P. Bindloss, E. Fradkin, V. Oganesyan, J. M. Tranquada, A. Kapitulnik, and C. Howald, Rev. Mod. Phys. 75, 1201 (2003).

${ }^{13}$ S. Sachdev, Rev. Mod. Phys. 75, 913 (2003).

${ }^{14}$ M. Vojta, Adv. Phys. 58, 699 (2009).

${ }^{15}$ P. Chandra, P. Coleman, and A. I. Larkin, Phys. Rev. Lett. 64, 88 (1990).

${ }^{16}$ L. Capriotti and S. Sachdev, Phys. Rev. Lett. 93, 257206 (2004).

${ }^{17}$ N. Read and S. Sachdev, Phys. Rev. Lett. 66, 1773 (1991).

${ }^{18}$ S. Sachdev and N. Read, Int. J. Mod. Phys. B 5, 219 (1991).

${ }^{19}$ H. Yamase and H. Kohno, J. Phys. Soc. Jpn. 69, 2151 (2000).

${ }^{20}$ C. J. Halboth and W. Metzner, Phys. Rev. Lett. 85, 5162 (2000).

${ }^{21}$ V. Oganesyan, S. A. Kivelson, and E. Fradkin, Phys. Rev. B 64, 195109 (2001).

${ }^{22}$ W. Metzner, D. Rohe, and S. Andergassen, Phys. Rev. Lett. 91, 066402 (2003).

${ }^{23}$ H.-Y. Kee, E. H. Kim, and C.-H. Chung, Phys. Rev. B 68 , 245109 (2003).

${ }^{24}$ H. Yamase, V. Oganesyan, and W. Metzner, Phys. Rev. B 72, 035114 (2005).

${ }^{25}$ L. Dell' Anna and W. Metzner, Phys. Rev. B 73, 045127 (2006); Phys. Rev. Lett. 98, 136402 (2007).

${ }^{26}$ J. Rech, C. Pepin, and A. V. Chubukov, Phys. Rev. B 74, 195126
(2006).

${ }^{27}$ M. J. Lawler, D. G. Barci, V. Fernandez, E. Fradkin, and L. Oxman, Phys. Rev. B 73, 085101 (2006).

${ }^{28}$ M. J. Lawler and E. Fradkin, Phys. Rev. B 75, 033304 (2007).

${ }^{29}$ P. Jakubczyk, P. Strack, A. A. Katanin, and W. Metzner, Phys. Rev. B 77, 195120 (2008).

${ }^{30}$ M. Zacharias, P. Wölfle, and M. Garst, Phys. Rev. B 80, 165116 (2009).

${ }^{31}$ D. Maslov and A. Chubukov, arXiv:0911.1251 (unpublished).

${ }^{32}$ H. v. Löhneysen, A. Rosch, M. Vojta, and P. Wölfle, Rev. Mod. Phys. 79, 1015 (2007).

${ }^{33}$ M. Vojta, Y. Zhang, and S. Sachdev, Phys. Rev. Lett. 85, 4940 (2000); 100, 089904(E) (2008).

${ }^{34}$ M. Vojta, Y. Zhang, and S. Sachdev, Int. J. Mod. Phys. B 14, 3719 (2000).

${ }^{35}$ E.-A. Kim, M. J. Lawler, P. Oreto, S. Sachdev, E. Fradkin, and S. A. Kivelson, Phys. Rev. B 77, 184514 (2008).

${ }^{36}$ Y. Huh and S. Sachdev, Phys. Rev. B 78, 064512 (2008).

${ }^{37}$ J. Polchinski, Nucl. Phys. B 422, 617 (1994).

${ }^{38}$ B. L. Altshuler, L. B. Ioffe, and A. J. Millis, Phys. Rev. B 50, 14048 (1994).

${ }^{39}$ S.-S. Lee, Phys. Rev. B 78, 085129 (2008).

${ }^{40}$ S.-S. Lee, Phys. Rev. B 80, 165102 (2009).

${ }^{41}$ A. Luther, Phys. Rev. B 19, 320 (1979).

${ }^{42}$ A. Houghton and J. B. Marston, Phys. Rev. B 48, 7790 (1993).

${ }^{43}$ H.-J. Kwon, A. Houghton, and J. B. Marston, Phys. Rev. Lett. 73, 284 (1994).

${ }^{44}$ F. Haldane, in Perspectives in Many-Particle Physics, Proceedings of the International School of Physics "Enrico Fermi" Course CXXI, edited by R. A. Broglia and J. R. Schrieffer (North-Holland, Amsterdam, 1994).

${ }^{45}$ A. H. Castro Neto and E. H. Fradkin, Phys. Rev. B 51, 4084 (1995).

${ }^{46}$ M. A. Metlitski and S. Sachdev, following paper, Phys. Rev. B 82, 075128 (2010).

${ }^{47}$ M. E. Simon and C. M. Varma, Phys. Rev. Lett. 89, 247003 (2002).

${ }^{48}$ E. Berg, C.-C. Chen, and S. A. Kivelson, Phys. Rev. Lett. 100, 027003 (2008).

${ }^{49}$ B. I. Halperin, P. A. Lee, and N. Read, Phys. Rev. B 47, 7312 (1993).

${ }^{50}$ Y. B. Kim, A. Furusaki, X.-G. Wen, and P. A. Lee, Phys. Rev. B 50, 17917 (1994).

${ }^{51}$ C. Nayak and F. Wilczek, Nucl. Phys. B 417, 359 (1994); 430, 534 (1994).

${ }^{52}$ Y.-B. Kim, P. A. Lee, and X.-G. Wen, Phys. Rev. B 52, 17275 (1995).

${ }^{53}$ A. Stern and B. I. Halperin, Phys. Rev. B 52, 5890 (1995).

${ }^{54}$ A. Stern, B. I. Halperin, F. von Oppen, and S. H. Simon, Phys. 
Rev. B 59, 12547 (1999).

${ }^{55}$ R. Shankar and G. Murthy, Phys. Rev. Lett. 79, 4437 (1997).

${ }^{56}$ N. Read, Phys. Rev. B 58, 16262 (1998).

${ }^{57}$ M. Hermele, T. Senthil, M. P. A. Fisher, P. A. Lee, N. Nagaosa, and X.-G. Wen, Phys. Rev. B 70, 214437 (2004).

${ }^{58}$ T. Senthil, Phys. Rev. B 78, 035103 (2008); 78, 045109 (2008).

${ }^{59}$ R. K. Kaul, Y. B. Kim, S. Sachdev, and T. Senthil, Nat. Phys. 4, 28 (2008).

${ }^{60}$ S. Sachdev, M. A. Metlitski, Y. Qi, and C. Xu, Phys. Rev. B 80, 155129 (2009).
${ }^{61}$ Y. Qi and S. Sachdev, Phys. Rev. B 81, 115129 (2010).

${ }^{62}$ S.-S. Lee, Phys. Rev. D 79, 086006 (2009).

${ }^{63}$ M. Cubrovic, J. Zaanen, and K. Schalm, Science 325, 439 (2009).

${ }^{64}$ H. Liu, J. McGreevy, and D. Vegh, arXiv:0903.2477 (unpublished); T. Faulkner, H. Liu, J. McGreevy, and D. Vegh, arXiv:0907.2694 (unpublished).

${ }^{65}$ F. Denef, S. A. Hartnoll, and S. Sachdev, Phys. Rev. D 80, 126016 (2009); S. Hartnoll and D. Hofman, arXiv:0912.0008 (unpublished). 